\title{
Polymorphic Nodal Elements and their Application in Discontinuous Galerkin Methods
}

\author{
Gregor J. Gassner ${ }^{\mathrm{a}}$ Frieder Lörcher ${ }^{\mathrm{a}}$ Claus-Dieter Munz ${ }^{\mathrm{a}}$ \\ Jan S. Hesthaven ${ }^{b}$ \\ ${ }^{\mathrm{a}}$ Institute for Aerodynamics and Gasdynamics \\ University of Stuttgart, Pfaffenwaldring 21, 70550 Stuttgart, Germany \\ ${ }^{\mathrm{b}}$ Division of Applied Mathematics \\ Brown University, Box F, Providence, RI 02912, USA
}

\begin{abstract}
In this work we discuss two different but related aspects of the development of efficient discontinuous Galerkin methods on hybrid element grids for the computational modeling of gas dynamics in complex geometries or with adapted grids. In the first part, a recursive construction of different nodal sets for $h p$ finite elements is presented. They share the property that the nodes along the sides of the two-dimensional elements and along the edges of the three-dimensional elements are the Legendre-Gauss-Lobatto points. The different nodal elements are evaluated by computing the Lebesgue constants of the corresponding Vandermonde matrix. In the second part, we apply these nodal elements as the basis for discontinuous Galerkin schemes. We use a modal based formulation and introduce a nodal based integration technique to reduce computational cost. We illustrate the performance of the scheme on several large scale applications and discuss its use in a recently developed space-time expansion discontinuous Galerkin scheme.
\end{abstract}

Key words: discontinuous Galerkin, nodal, modal, polynomial interpolation, $h p$ finite elements, Lebesgue constants, quadrature free, unstructured, triangle, quadrilateral, polygonal, tetrahedron, hexahedron, prism, pentahedron, pyramid.

Email addresses: gassner@iag. uni-stuttgart . de (Gregor J. Gassner), loercher@iag. uni-stuttgart. de (Frieder Lörcher), munz@iag. uni-stuttgart. de (Claus-Dieter Munz), Jan. Hesthaven@Brown. edu (Jan S. Hesthaven). 


\section{Introduction}

While discontinuous Galerkin (DG) methods were first proposed in the early 1970's in [35] it was not until the more recent development, initiated in the work of Cockburn and Shu $[10,11,9,7,12]$, that these methods matured into a powerful computational tool for the solution of systems of conservation laws and the equations of gas dynamics $[13,4]$. The extension to problems of viscous gas dynamics was initiated in $[3,5]$ and this again has lead to several related formulations $[28,34,16]$ for the compressible Navier-Stokes equations. Many examples and further details along these lines can be found in $[8,29]$ and [25].

In spite of these significant advances over the last decade, discontinuous Galerkin methods still suffer from being perceived as being too expensive when compared to more traditional methods such as finite volume methods. This is particularly true for viscous problems, where the common solution approach is based on a mixed finite element formulation, which was introduced in [3] and extended to higher order problems in $[38,39]$. In recent developments for the DG discretization of second order terms $[14,15,30]$, the introduction of auxiliary variables is circumvented by the use of two partial integrations, or by multiple partial integrations for higher order operators [6].

Apart from this, however, a major computational cost is found in the traditional use of full order integration in the basic implementation, leading to excessive computational cost for nonlinear problems. Deriving inspiration from the classic spectral methods [21] it is natural to consider the use of a nodal basis, leading to a formulation which in spirit shares much with a spectral collocation formulation in which the boundary conditions are imposed weakly. Such methods, often known as spectral penalty methods, have been developed for the compressible Navier-Stokes equations in [17-19] and extended to non-tensorial elements in [20,22].

The main advantages of such a formulation are found in the exact reduction to the standard discontinuous Galerkin formulation for linear problems, hence ensuring the accuracy for smooth problems, and the quadrature free approach for nonlinear problems, leading to a dramatic reduction in the overall computational cost. Furthermore, the use of a nodal basis with the correct structure of the points along the edges and faces leads to a natural separation of the basis into boundary and internal degrees of freedom. This becomes particularly beneficial for schemes using a high-order basis. As is usually the case, all good things comes with a price and in this case the loss of exact integration opens the possibility for instabilities driven by aliasing. This is, however, a well known phenomenon and is well understood within the community of spectral methods [21]. We shall return to this concern briefly later.

One of the limitations of past nodal based formulations and schemes has been the 
reliance on either cubic or tetrahedral element shapes. While these suffice in many cases, for problems with significant geometric flexibility one is tempted to also use more general types of elements such as prisms and pyramids.

In this work we explore how one construct such nodal general elements, using a recursive construction, and optimize these for maximum accuracy by minimizing the Lebesgue constant of the associated multivariate Lagrange polynomial. This is discussed in Section 2 and sets the stage for Section 3 where we discuss in detail the use of these general elements in a discontinuous Galerkin scheme and return to the issues of aliasing and instabilities caused by this. We shall also discuss how nodal elements can be used with advantage in an already existing scheme based on a modal expansion and finally we use the recently developed explicit spacetime discretization to arrive at the fully discrete explicit scheme. In Section 4 we demonstrate how this general scheme, employing polymorphic elements and local time-stepping, can be used with benefit for both linear and nonlinear wave problems and, finally, the full three-dimensional compressible Navier-Stokes equations. Most of the tests illustrate the potential for a 4 fold reduction in computational time without impacting the accuracy by using the nodal based approach for large scale simulations. Section 5 concludes with a few general remarks and outlook toward future work.

\section{The nodal elements}

We will first focus on defining different sets of high order basis functions for a given grid cell $Q \subset \mathbb{R}^{d}$. We introduce the monomial basis $\left\{\pi_{i}\right\}_{i=1, \ldots, N}$ for the space of polynomials with degree less than or equal than $p$, where every basis function $\pi_{i}$ could be written as

$$
\pi_{i}(\vec{x})=x_{1}^{\alpha_{1}^{i}} \cdot \ldots \cdot x_{d}^{\alpha_{d}^{i}} \text { with } 0 \leq \alpha_{1}^{i}+\ldots+\alpha_{d}^{i} \leq p .
$$

The dimension $N$ of this space depends on the order $p$ and on the spatial dimension $d$ of the grid cell $Q$ and is given by

$$
N=N(p, d)=\frac{(p+d) !}{d ! p !} .
$$

Based on the monomial basis $\left\{\pi_{i}\right\}_{i=1, \ldots, N}$ and the geometry of the grid cell $Q$ the construction of an orthonormal basis $\left\{\varphi_{i}\right\}_{i=1, \ldots, N}$ using Gram-Schmidt orthogonalization is straight forward. This basis set is characterized by the property

$$
\int_{Q} \varphi_{i}(\vec{x}) \varphi_{j}(\vec{x}) d \vec{x}=\delta_{i j},
$$

which holds for arbitrary grid cell shapes. With this modal basis we are now able to define a set of nodal basis functions. Given a set of interpolation points 
$\left\{\vec{\xi}_{j}\right\}_{j=1, \ldots, M_{I}} \subset Q$, we can construct the nodal Lagrange basis $\left\{\psi_{j}\right\}_{j=1, \ldots, M_{I}}$ defined by the conditions

$$
\begin{aligned}
& \psi_{j}\left(\vec{\xi}_{i}\right)=\delta_{i j}, \\
& u(\vec{x}):=\sum_{j=1}^{N} \hat{u}_{j} \varphi_{j}(\vec{x}) \stackrel{!}{=} \sum_{i=1}^{M_{I}} \widetilde{u}_{i} \psi_{i}(\vec{x}) .
\end{aligned}
$$

Combining these conditions yields the transformations

$$
\underline{\underline{V}} \underline{\hat{u}}=\underline{\widetilde{u}} \text { and } \underline{\underline{V}}^{T} \underline{\psi}=\underline{\phi},
$$

where we introduce the generalized Vandermonde matrix $\underline{\underline{V}}$ with entries

$$
V_{i j}=\varphi_{j}\left(\vec{\xi}_{i}\right), \quad i=1, \ldots, M_{I} ; j=1, \ldots, N
$$

The inverse of the Vandermonde matrix is not uniquely defined as $M_{I} \neq N$. If one is interested in avoiding this problem, one has to extend the modal basis from dimension $N$ to dimension $M_{I}$. We refer to Lörcher and Munz [32] for a strategy to find a basis extensions for non-tensor product interpolation on a cartesian grid. However, the extension of this approach to the general case is not straightforward, as the non-singularity of the Vandermonde matrix is not guaranteed. To overcome this issue a singular value decomposition based strategy is used to define the following (pseudo) inverse transformations

$$
\underline{\hat{u}}=\underline{\underline{V}}^{-1} \underline{\widetilde{u}} \text { and } \underline{\psi}=\underline{\underline{V}}^{-T} \underline{\phi} .
$$

Using the pseudo-inverse Vandermonde matrix, condition (4) is only satisfied in the least squares sense. Thus, if we define the polynomial approximation of a function $f$ as

$$
f(\vec{x}) \approx f_{I}(\vec{x}):=\sum_{j=1}^{M_{I}} f\left(\vec{\xi}_{j}\right) \psi_{j}(\vec{x})=: \underline{\psi}^{T} \underline{\tilde{f}},
$$

the nodal degree of freedom $\tilde{f}_{j}=f\left(\vec{\xi}_{j}\right)$ is not the value of the interpolation $f_{I}(\vec{x})$ at the node $\vec{x}=\vec{\xi}_{j}$, as $\psi_{j}\left(\vec{\xi}_{i}\right) \neq \delta_{i j}$. Furthermore, the modal approximation

$$
f(\vec{x}) \approx f_{M}(\vec{x}):=\sum_{j=1}^{N} \hat{f}_{j} \phi_{j}(\vec{x}) \text { with } \underline{\hat{f}}=\underline{\underline{V}}^{-1} \underline{\tilde{f}}
$$

is in the general case not equal to the nodal approximation

$$
f_{I}(\vec{x}) \neq f_{M}(\vec{x})
$$

A good measure of the quality of such a polynomial approximation is given by the Lebesgue constant $\Lambda$, defined as

$$
\Lambda:=\max _{\vec{x} \in Q} \sum_{j=1}^{M_{I}}\left|\psi_{j}(\vec{x})\right| .
$$


With this definition one easily realizes that

$$
\left\|f-f_{I}\right\|_{\infty} \leq(1+\Lambda)\left\|f-f^{*}\right\|_{\infty}
$$

where $\|.\|_{\infty}$ is the usual maximum norm and $f^{*}$ is the best approximating polynomial of $f$. As the nodal basis $\left\{\psi_{j}\right\}_{j=1, \ldots, M_{I}}$ depends only on the interpolation points $\left\{\vec{\xi}_{i}\right\}_{i=1, \ldots, M_{I}}$, we next focus on the construction of nodal sets for different grid cell shapes which minimize the growth of the Lebesgue constant with order $p$. We restrict the attention to sets of interpolation points $\Omega_{I}:=\left\{\vec{\xi}_{i}\right\}_{i=1, \ldots, M_{I}}$ with the following characteristics

- the interpolation based on these points is of order $p$ for functions defined in the volume and for functions defined on the grid cell surfaces. This guarantees that the basis separates into boundary and interior components.

- the distribution of the points reflects the possible symmetries of the grid cell,

- the size of the nodal set $M_{I} \geq N$ depends on the order $p$, the dimension $d$ and the shape of the grid cell.

\subsection{One-dimensional node distributions}

For an interval, $p+1$ points have to be chosen. There may be a number of different distributions of the $p+1$ points with the restriction that the endpoints are included. For instance, one can choose equidistant (E) points, Chebychef-GaussLobatto points or Legendre-Gauss-Lobatto (LGL) points. We choose for every side in $2 \mathrm{D}$ and edge in $3 \mathrm{D}$ the LGL node distribution, as these are known for a good Lebesgue constant $\Lambda$. An extended discussion of the one-dimensional case can be found in [23]. Based on the LGL node distribution we define the following warp function for $x \in[0 ; 1]$

$$
w_{p}(x)=\sum_{j=1}^{p+1}\left(\xi_{j}^{L G L}-\xi_{j}^{E}\right) \psi_{j}^{E}(x),
$$

where $\left\{\xi_{j}^{L G L}\right\}_{j=1, \ldots, p+1}$ are the Legendre-Gauss-Lobatto points, $\left\{\xi_{j}^{E}\right\}_{j=1, \ldots, p+1}$ denote the equidistant points and $\left\{\psi_{j}^{E}\right\}_{j=1, \ldots, p+1}$ the Lagrange polynomials based on the equidistant points. According to [25], $w_{p}(x)$ is a $(p+1)$ th order approximation to the function which maps the 'bad' points (E) to the 'good' points (LGL).

\subsection{Two-dimensional node distributions}

In two space dimensions we split the set of interpolation points $\Omega_{I}(p)$ into two parts: The set of points that live in the interior of the cell and the set of points that live on the surface, named $\Omega_{I}^{S}(p)$. The set $\Omega_{I}^{S}(p)$ is defined such that it contains 
$p+1$ LGL points for each side of the grid cell surface. This guarantees that the nodal approximation on the whole surface is of order $p+1$ and a separated basis by polynomial uniqueness. We note that using only these surface points for the approximation within the volume, the corresponding Vandermonde matrix is nonsingular for $p$ up to a value $p^{*}$, which depends on the shape of the grid cell. The value for $p^{*}$ is 2 and 3 for triangles and quadrilaterals, respectively. Hence, for an interpolation with $p>p^{*}$, additional points in the interior of the grid cell are needed. The definition of these interpolation points can be done in the following recursive way

$$
\Omega_{I}(p):= \begin{cases}\emptyset & \text { for } p<0, \\ \left\{\vec{x}_{\text {bary }}\right\} & \text { for } p=0, \\ \mathcal{M}_{r}\left(\Omega_{I}^{S}(p)\right) \cup \Omega_{I}\left(p-p^{*}+\pi_{2 D}\right) & \text { for } p>0 .\end{cases}
$$

We notice that the interior nodes consist of nested and 'shrinked' surface points. The mapping $\mathcal{M}_{r}$ determines how the point sets are nested and shrunk for every recursion step $r$, e.g., the mapping for the first recursion $r=0$ is the identity, as the first points of the set $\Omega_{I}^{S}(p)$ are lying on the real surface of the grid cell and thus will not be shrunk. A simple approach for the mappings $\mathcal{M}_{r}$ for $r>0$ would be one which yields an equidistant nesting. However, it is well known that the Lebesgue constant of the corresponding nodal basis is improved, when the node distribution is more dense close to the boundary of the grid cell. Thus, to improve the nodal set we propose to use a mapping which yields LGL-type nesting. In this work the warp function (13) is used to define the following barycentric mapping

$$
\begin{aligned}
\mathcal{M}_{r}(\vec{\xi}) & =\left(\vec{\xi}-\vec{x}_{\text {bary }}\right) \alpha(r)+\vec{x}_{\text {bary }}, \\
\alpha(r) & =1-2 w_{p}(r / \widetilde{r})
\end{aligned}
$$

where $\vec{\xi} \in \Omega_{I}^{S}(p), \vec{x}_{\text {bary }}$ denotes the barycenter of the grid cell and $r$ the recursion level. $\widetilde{r}$ is two times the maximum number of recursions $\widetilde{r}=2 r_{\text {max }}$. We subtract one from $\widetilde{r}$, if the innermost interpolation point set consists only of the grid cell barycenter $\tilde{r}=2 r_{\max }-1$. Another approach is to start with the pure equidistant point distribution and optimize the nodal set with electrostatic considerations, as proposed by Hesthaven [23]. To illustrate these different strategies, we plot the corresponding node distributions of the $p=9\left(\pi_{2 D}=0\right)$ quadrilateral in figure 1 . The set with a purely equidistant distribution yields a Lebesgue constant $\Lambda=97$, whereas the LGL points with equidistant nesting yields $\Lambda=44$. Using LGL points and LGL-type nesting yields a Lebesgue constant of 21 , which is slightly greater than $\Lambda=17$ for the electro-static optimized points. Although the electro-static optimized interpolation points yield the best Lebesgue constant, we use the LGL points with LGL-type nesting in the computations shown below, as these point sets are easily and straight forward to implement. An important parameter in the recursion formula (14) is the integer $\pi_{2 D}$ which can be used to tune the relation between the interpolation quality and number of points. For $\pi_{2 D}=0$, as considered up to now, algorithm (14) yields the smallest possible number of points and thus, the 

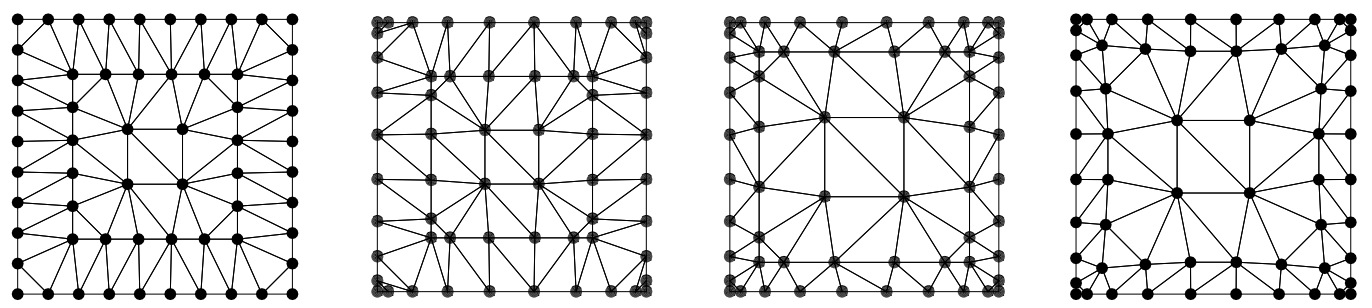

Fig. 1. Quadrilateral with $p=9(\pi=0)$. From left to right: pure equidistant distribution, LGL points with equidistant nesting, LGL points with LGL-type nesting and optimized points.

most efficient scheme according to the computational effort. However, we observed that in some cases, especially for quadrilaterals, the use of a few more points pays off in terms of a dramatically improved accuracy. The parameter $\pi_{2 D}$ with $0 \leq$ $\pi_{2 D} \leq p^{*}-1$ can be used to control the number of recursions in (14). Figure 2 shows the ratio of the overall interpolation points $M_{I}(p)$ and the number $N(p)$ of the basis functions as a function of the polynomial degree $p$ for different values of $\pi_{2 D}$. The plot indicates that for triangles and $\pi_{2 D}=0$ the number is always optimal. For quadrilaterals and $\pi_{2 D}=0$ the number of interpolation points converges to the optimum with increasing $p$. In all the calculations presented in the following we
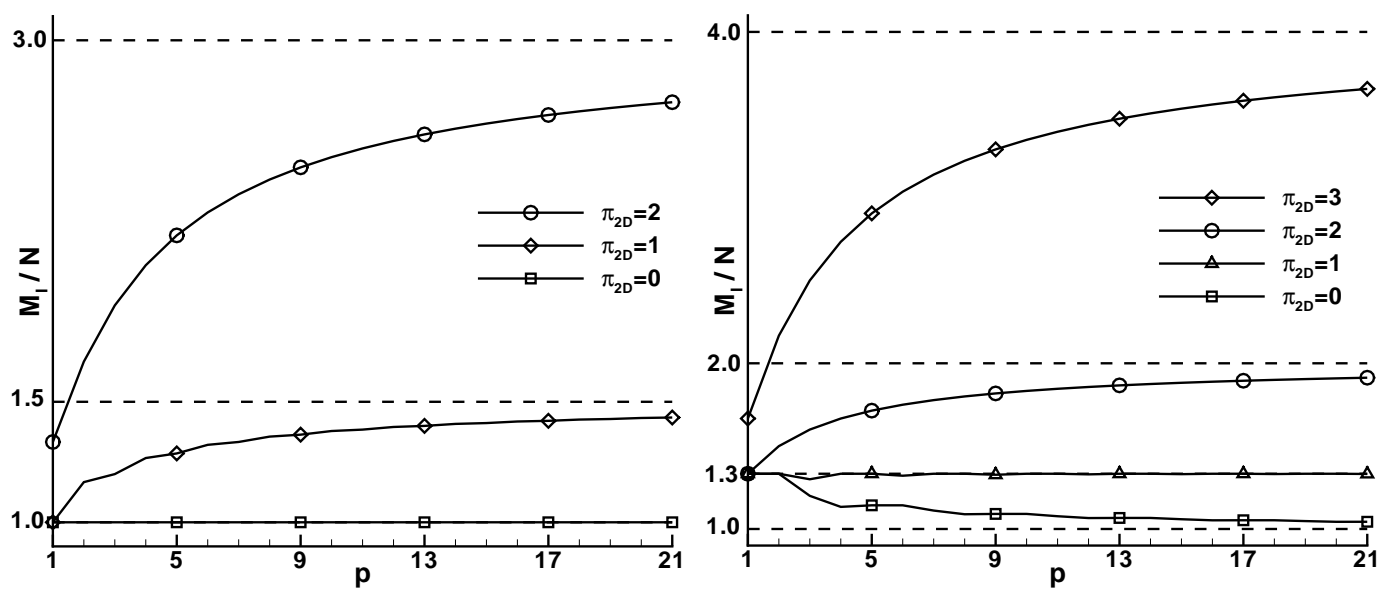

Fig. 2. Ratio of the number $M_{I}(p)$ of interpolation points and the dimension $N(p)$ of the polynomial space as a function of the polynomial degree $p$ for different parameters $\pi_{2 D}$, left for triangles and right for quadrilaterals. The limits for $p \rightarrow \infty$ are indicated with a dashed line.

use $\pi_{2 D}=0$ for triangles and $\pi_{2 D} \in\{0,1\}$ for quadrilaterals. For this type of interpolation points the corresponding Lebesgue constants $\Lambda$ are listed in table 1 .

\subsection{Three-dimensional node distributions}

The definition of the three-dimensional set of interpolation points is done analogously to that of the two-dimensional case. Again, the set $\Omega_{I}(p)$ is split into two 
Table 1

\begin{tabular}{c|cc|cc|ll}
$p$ & $M_{I}$ & $\Lambda$ & $M_{I}$ & $\Lambda$ & $M_{I}$ & $\Lambda$ \\
& $\operatorname{tri}\left(\pi_{2 D}=0\right)$ & \multicolumn{2}{|l}{ quad $\left(\pi_{2 D}=0\right)$} & \multicolumn{2}{l}{ quad $\left(\pi_{2 D}=1\right)$} \\
\hline 1 & 3 & 1.0 & 4 & 1.5 & 4 & 1.5 \\
2 & 6 & 1.7 & 8 & 3.0 & 8 & 3.0 \\
3 & 10 & 2.1 & 12 & 4.0 & 13 & 3.2 \\
4 & 15 & 3.8 & 17 & 4.2 & 20 & 5.3 \\
5 & 21 & 3.2 & 24 & 5.8 & 28 & 4.6 \\
6 & 28 & 4.6 & 32 & 7.5 & 37 & 4.5 \\
7 & 36 & 6.8 & 40 & 15.3 & 48 & 5.1 \\
8 & 45 & 7.5 & 49 & 14.5 & 60 & 7.5 \\
9 & 55 & 8.6 & 60 & 21.0 & 73 & 8.0 \\
10 & 66 & 11.2 & 72 & 28.6 & 88 & 10.8 \\
11 & 78 & 18.8 & 84 & 61.8 & 104 & 14.8 \\
12 & 91 & 20.2 & 97 & 62.7 & 121 & 15.4 \\
\hline
\end{tabular}

Lebesgue constants $\Lambda$ and number of interpolation points $M_{I}$ for the two-dimensional interpolation points.

parts, where $\Omega_{I}^{S}\left(p, \pi_{2 D}\right)$ denotes the set of points on the surface. The recursion algorithm reads as follows

$$
\Omega_{I}(p):= \begin{cases}\emptyset & \text { for } p<0, \\ \left\{\vec{x}_{\text {bary }}\right\} & \text { for } p=0, \\ \mathcal{M}_{r}\left(\Omega_{I}^{S}\left(p, \pi_{2 D}\right)\right) \cup \Omega_{I}\left(p-p^{*}+\pi_{3 D}\right) & \text { for } p>0 .\end{cases}
$$

In this work the $3 D$ standard shapes, namely tetrahedra, hexahedra, pentahedra (prisms) and pyramids are considered. The surfaces of this standard grid cells consist of triangles and quadrilaterals. Thus, for the definition of the surface point set $\Omega_{I}^{S}\left(p, \pi_{2 D}\right)$ we can use the two-dimensional nodal points from the previous subsection. Again, using surface points only yields non-singular interpolation up to a polynomial degree $0<p \leq p^{*}$. The value of $p^{*}$ is 3 for the tetrahedron, 5 for the hexahedron and 4 for the pentahedron and pyramid, respectively. We note that these values are independent of the choice of the parameter $\pi_{2 D}$. Although the number of surface points increases with greater $\pi_{2 D}$, the rank of the volume interpolation does not. We thus use the recursive nesting strategy (16) and introduce an additional parameter $\pi_{3 D}$, which controls the number of recursions. The mapping $\mathcal{M}_{r}$ is again used to shrink the new nested surface points in a LGL-type manner (15). In figure 3 the ratios of the interpolation points $M_{I}(p)$ between the optimal number $N(p)$ for different parameters $\pi:=\left(\pi_{3 D}, \pi_{2 D}\right)$ are plotted. Again for tetrahedra and $\pi=(0,0)$ the number of interpolation points are always optimal, whereas for other 
grid cell shapes the ratio converges to 1.0 for $p \rightarrow \infty$. Compared to the $2 D$ case the convergence for the $3 D$ case is slower, however the magnitudes of the ratios are still reasonable. The corresponding Lebesgue constants are listed in tables 2 and 3.
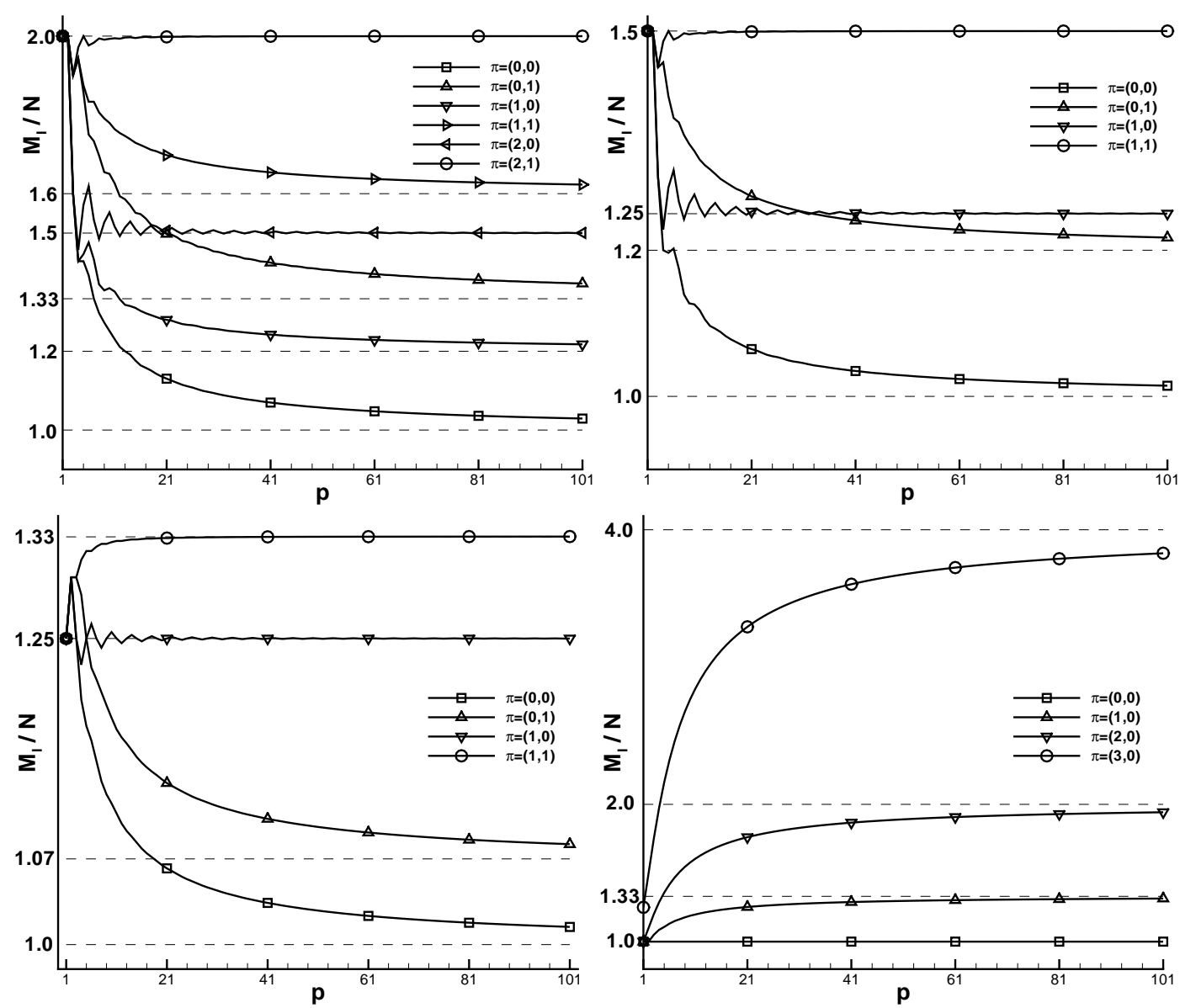

Fig. 3. Ratio of the number $M_{I}(p)$ of interpolation points and the dimension $N(p)$ of the polynomial space as a function of the polynomial degree $p$ for hexahedron (top left), pentahedron (top right), pyramid (bottom left) and tetrahedron (bottom right) and different parameters $\pi=\left(\pi_{3 D}, \pi_{2 D}\right)$. The limits for $p \rightarrow \infty$ are indicated with dashed lines. 


\begin{tabular}{c|c|c|c|c|c|c|c|c|c|c|c}
\hline \hline tetrahedron $/ p$ & 1 & 2 & 3 & 4 & 5 & 6 & 7 & 8 & 9 & 10 & 11 \\
\hline$M_{I}$ & 4 & 10 & 20 & 35 & 56 & 84 & 120 & 165 & 220 & 286 & 364 \\
$\pi=(0,0) / \Lambda$ & 1.0 & 2.0 & 2.9 & 4.0 & 6.4 & 7.9 & 10.8 & 17.6 & 22.0 & 34.8 & 36.5 \\
\hline \hline hexahedron $/ p$ & 1 & 2 & 3 & 4 & 5 & 6 & 7 & 8 & 9 & 10 & 11 \\
\hline$M_{I}$ & 8 & 20 & 32 & 50 & 80 & 117 & 160 & 214 & 280 & 358 & 448 \\
$\pi=(0,0) / \Lambda$ & 1.5 & 5.0 & 6.4 & 8.8 & 17.0 & 20.3 & 41.5 & 47.6 & 103.6 & 201.3 & 454.2 \\
\hline$M_{I}$ & 8 & 20 & 32 & 50 & 81 & 124 & 172 & 226 & 298 & 389 & 492 \\
$\pi=(1,0) / \Lambda$ & 1.5 & 5.0 & 6.4 & 8.8 & 11.6 & 35.6 & 37.1 & 46.6 & 103.2 & 113.5 & 148.2 \\
\hline$M_{I}$ & 8 & 20 & 38 & 68 & 104 & 147 & 208 & 280 & 364 & 472 & 592 \\
$\pi=(0,1) / \Lambda$ & 1.5 & 5.0 & 4.8 & 15.6 & 11.2 & 13.0 & 30.4 & 32.7 & 52.0 & 111.6 & 323.5 \\
\hline$M_{I}$ & 8 & 20 & 38 & 68 & 105 & 154 & 220 & 298 & 394 & 509 & 642 \\
$\pi=(1,1) / \Lambda$ & 1.5 & 5.0 & 4.8 & 15.6 & 8.9 & 18.1 & 13.3 & 31.0 & 49.4 & 58.0 & 78.0 \\
\hline$M_{I}$ & 8 & 20 & 32 & 51 & 88 & 136 & 184 & 245 & 336 & 444 & 552 \\
$\pi=(2,0) / \Lambda$ & 1.5 & 5.0 & 6.4 & 7.8 & 9.1 & 14.0 & 30.2 & 28.3 & 40.2 & 56.9 & 124.2 \\
\hline$M_{I}$ & 8 & 20 & 38 & 69 & 112 & 166 & 238 & 329 & 438 & 570 & 726 \\
$\pi=(2,1) / \Lambda$ & 1.5 & 5.0 & 4.8 & 5.9 & 8.9 & 11.1 & 12.5 & 20.3 & 21.2 & 31.5 & 61.2 \\
\hline$T a b 2$
\end{tabular}

Table 2

Lebesgue constants $\Lambda$ and number of interpolation points $M_{I}$ for the $3 D$ interpolation sets with different parameters $\pi=\left(\pi_{3 D}, \pi_{2 D}\right)$. 


\begin{tabular}{c|c|c|c|c|c|c|c|c|c|c|c}
\hline \hline pentahedron $/ p$ & 1 & 2 & 3 & 4 & 5 & 6 & 7 & 8 & 9 & 10 & 11 \\
\hline$M_{I}$ & 6 & 15 & 26 & 42 & 67 & 101 & 141 & 188 & 248 & 322 & 407 \\
$\pi=(0,0) / \Lambda$ & 1.7 & 3.7 & 4.4 & 6.0 & 8.1 & 21.4 & 22.7 & 42.3 & 96.7 & 112.1 & 175.2 \\
\hline$M_{I}$ & 6 & 15 & 26 & 43 & 72 & 110 & 152 & 205 & 278 & 365 & 458 \\
$\pi=(1,0) / \Lambda$ & 1.7 & 3.7 & 4.4 & 5.9 & 10.0 & 11.2 & 23.4 & 24.2 & 61.6 & 74.2 & 167.8 \\
\hline$M_{I}$ & 6 & 15 & 29 & 51 & 79 & 116 & 165 & 224 & 296 & 382 & 482 \\
$\pi=(0,1) / \Lambda$ & 1.7 & 3.7 & 4.1 & 9.4 & 7.2 & 15.6 & 15.0 & 34.2 & 70.8 & 86.8 & 117.6 \\
\hline$M_{I}$ & 6 & 15 & 29 & 52 & 84 & 125 & 179 & 247 & 329 & 428 & 545 \\
$\pi=(1,1) / \Lambda$ & 1.7 & 3.7 & 4.1 & 5.7 & 10.0 & 8.7 & 13.0 & 17.2 & 33.3 & 34.5 & 60.2 \\
\hline \hline pyramid/p & 1 & 2 & 3 & 4 & 5 & 6 & 7 & 8 & 9 & 10 & 11 \\
\hline$M_{I}$ & 5 & 13 & 25 & 42 & 66 & 98 & 138 & 187 & 247 & 319 & 403 \\
$\pi=(0,0) / \Lambda$ & 1.5 & 3.0 & 4.2 & 6.8 & 9.7 & 15.6 & 24.5 & 39.7 & 71.4 & 146.9 & 366.2 \\
\hline$M_{I}$ & 5 & 13 & 25 & 43 & 70 & 106 & 150 & 205 & 275 & 359 & 455 \\
$\pi=(1,0) / \Lambda$ & 1.5 & 3.0 & 4.2 & 5.3 & 7.2 & 11.4 & 20.0 & 20.8 & 54.8 & 38.6 & 83.6 \\
\hline$M_{I}$ & 5 & 13 & 26 & 45 & 70 & 103 & 146 & 199 & 263 & 339 & 428 \\
$\pi=(0,1) / \Lambda$ & 1.5 & 3.0 & 3.8 & 8.4 & 9.0 & 13.1 & 20.2 & 32.8 & 65.5 & 137.7 & 360.6 \\
\hline$M_{I}$ & 5 & 13 & 26 & 46 & 74 & 111 & 159 & 219 & 292 & 380 & 484 \\
$\pi=(1,1) / \Lambda$ & 1.5 & 3.0 & 3.8 & 6.0 & 7.0 & 9.5 & 12.9 & 18.0 & 27.4 & 27.4 & 42.1 \\
\hline$T a b e$ & & & & & & & & \\
\hline
\end{tabular}

Table 3

Lebesgue constants $\Lambda$ and number of interpolation points $M_{I}$ for the $3 D$ interpolation sets with different parameters $\pi=\left(\pi_{3 D}, \pi_{2 D}\right)$. 


\section{Application in discontinuous Galerkin methods}

In the following we will discuss in detail how to construct a discontinuous Galerkin (DG) scheme using the nodal elements developed above. To keep matters simple we restrict the discussion to a scalar conservation law of the form

$$
u_{t}+\vec{\nabla} \cdot \vec{f}(u)=0
$$

with appropriate initial and boundary conditions in a domain $\Omega \times[0, T] \subset \mathbb{R}^{d} \times \mathbb{R}_{0}^{+}$. The base of the semi-discrete DG formulation is a local weak formulation, which is obtained for a grid cell $Q \subset \Omega$ by multiplying (17) by a test function $\phi=\phi(\vec{x})$ and integrating over $Q$

$$
\int_{Q}\left(u_{t}+\vec{\nabla} \cdot \vec{f}(u)\right) \phi d \vec{x}=0 .
$$

The usual weak formulation results after spatial integration by parts

$$
\int_{Q} u_{t} \phi d \vec{x}+\int_{\partial Q}(\vec{f}(u) \cdot \vec{n}) \phi d s-\int_{Q} \vec{f}(u) \cdot \vec{\nabla} \phi d \vec{x}=0 .
$$

For the DG discretization the exact solution $u$ is next replaced by a piecewise polynomial approximation $u_{h}$. As this approximation is in general discontinuous across grid cell interfaces, the surface flux integrals are not well defined. To get an unique solution and a stable discretization, the normal flux $\vec{f} \cdot \vec{n}$ in the surface integral is replaced with a numerical flux function $g_{\vec{n}}$, which depends on the values from both sides of the grid cell interface. Independent of the choice of the numerical flux $g_{\vec{n}}$, there are a lot of different ways of how to implement the semi-discrete DG scheme. The implementations differ in terms of 'evaluation of the integrals' and 'representation of the approximation $u_{h}$ '. Recently, Hesthaven and Warburton introduced the nodal DG scheme [24]. In their formulation, the approximation $u_{h}$ is represented using the nodal basis functions $\left\{\psi_{j}\right\}_{j=1, \ldots, M_{I}}$, which are furthermore chosen as test functions. In this work, we choose a more 'classic' approach, where we use the modal basis functions $\left\{\phi_{j}\right\}_{j=1, \ldots, N}$ to define the test functions and the DG polynomial

$$
u_{h}(\vec{x}, t):=\sum_{j=1}^{N} \hat{u}_{j}(t) \varphi_{j}(\vec{x}) \text { for } \vec{x} \in Q,
$$

with the time dependent modal DOF $\left\{\hat{u}_{j}(t)\right\}_{j=1, \ldots, N}$. In standard modal DG implementations, the evaluation of the integrals is usually done with Gauss integration. For instance we get the following approximation for the first volume integral

$$
\begin{aligned}
\int_{Q} f_{1}\left(u_{h}\right) \frac{\partial \underline{\varphi}}{\partial x_{1}}(\vec{x}) d \vec{x} & \approx \sum_{j=1}^{(p+1)^{d}} f_{1}\left(u_{h}\left(\vec{\chi}_{j}\right)\right) \frac{\partial \underline{\varphi}}{\partial x_{1}}\left(\vec{\chi}_{j}\right) \omega_{j}, \\
& =: \underline{\underline{K}}^{1, G P} \underline{f}_{1}
\end{aligned}
$$


where $\omega_{j}$ are the Gauss weights, $\vec{\chi}_{j}$ the Gauss positions, $\underline{f}_{1}$ the vector of flux evaluations and $\underline{\underline{K}}^{1, G P}$ the integration matrix with

$$
\left(\underline{\underline{K}}^{1, G P}\right)_{i j}:=\frac{\partial \varphi_{i}}{\partial x_{1}}\left(\vec{\chi}_{j}\right) \omega_{j}, i=1, \ldots, N ; j=1, \ldots,(p+1)^{d} .
$$

We note that $u_{h}\left(\vec{\chi}_{j}\right)$ is evaluated using (20). If we consider a hexahedron with a $p=5$ approximation, we get $(p+1)^{d}=216$ evaluations with this strategy for the approximation of the volume integrals. We will show in the next subsection how to make use of the nodal elements to reduce the computational complexity of modal implementations.

\subsection{The modal DG scheme with nodal integration}

We first introduce the nodal interpolation of the non-linear flux function according to $(8)$

$$
f_{1}\left(u_{h}(\vec{x})\right) \approx f_{1, I}(\vec{x}):=\sum_{i=1}^{M_{I}} \widetilde{f}_{1, i} \psi_{i}(\vec{x})
$$

where the nodal DOF is calculated as $\tilde{f}_{1, i}=f_{1}\left(u_{h}\left(\vec{\xi}_{i}\right)\right)$. The evaluation of the DG polynomial (20) at the nodal points can be done using the Vandermonde matrix (5)

$$
\underline{\widetilde{u}}=\underline{\underline{V}} \underline{\underline{u}},
$$

yielding the nodal DOF of the flux as $\widetilde{f}_{1, i}=f_{1}\left(\widetilde{u}_{i}\right)$. As a next step, the interpolation of the flux function is inserted into the volume integral and integrated exactly

$$
\begin{aligned}
\int_{Q} f_{1}\left(u_{h}\right) \frac{\partial \varphi_{j}}{\partial x_{1}}(\vec{x}) d \vec{x} & \approx \int_{Q} f_{1, I}(\vec{x}) \frac{\partial \varphi_{j}}{\partial x_{1}}(\vec{x}) d \vec{x}, \\
& =: \underline{\underline{K}}^{1} \underline{\tilde{f}}_{1},
\end{aligned}
$$

where we introduced the general stiffness matrix

$$
\underline{\underline{K}}^{1}:=\int_{Q} \frac{\partial \underline{\varphi}}{\partial x_{1}}(\vec{x}) \underline{\psi}^{T}(\vec{x}) d \vec{x}=\int_{Q} \frac{\partial \underline{\varphi}}{\partial x_{1}}(\vec{x}) \underline{\varphi}^{T}(\vec{x}) d \vec{x} \underline{\underline{V}}^{-1}=: \underline{\underline{K}}^{1, M} \underline{\underline{V}}^{-1}
$$

The evaluation of the stiffness matrix can be done with Gauss integration in an initial phase of the simulation, yielding a quadrature free approach. The surface integrals are treated in a similar manner. Comparing computational complexity we only need $M_{I}$ evaluations to calculate the volume integrals. Considering for instance the $p=5(\pi=(1,1))$ hexahedron we get $M_{I}=105$. Furthermore as the developed nodal elements support an interpolation in the volume and on the boundary at the same time no additional evaluations of the polynomial are needed to calculate the surface integrals. We note that the modal DG with nodal integration and the nodal DG [25] are strongly related. In fact the modal DG scheme with 
nodal integration can be interpreted as a nodal DG scheme using modal DOF and the Vandermonde matrix for the calculation of the nodal DOF (24). Reducing the accuracy of quadrature and relying on nodal products when computing nonlinear fluxes naturally introduces an error, known in spectral methods as aliasing [21]. However, the scheme maintains its full linear accuracy and the potential for aliasing driven instabilities is well understood and can, if needed, be controlled by the use of a weak modal filter (see [25]). In the present work, however, we have not found any need for this additional stabilization for any of the examples presented later.

\section{Computational examples and validations}

In the following we shall present a number of examples of increasing complexity to thoroughly validate the developed scheme. The spatial discontinuous Galerkin scheme is integrated in time using the recently developed space-time expansion (STE) approach [31,15], which allows a consistent arbitrary high order accurate local time stepping.

\subsection{Linear Wave Propagation}

In this subsection the spatial accuracy of the nodal integration approach for a linear problem is investigated. We use the linearized Euler equations (LEE) as a model problem for linear wave propagation

$$
U_{t}+\vec{\nabla} \cdot \vec{F}(U)=0
$$

with the vector of the conservative variables $U=\left(\rho^{\prime}, u^{\prime}, v^{\prime}, w^{\prime}, p^{\prime}\right)^{T}$ and the LEE fluxes $\vec{F}:=\left(F_{1}, F_{2}, F_{3}\right)^{T}:=\left(\underline{A}_{1} U, \underline{A}_{2} U, \underline{A}_{3} U\right)^{T}$ with the Jacobi matrices

$\underline{A}_{1}=\left(\begin{array}{ccccc}u_{0} & \rho_{0} & 0 & 0 & 0 \\ 0 & u_{0} & 0 & 0 & \frac{1}{\rho_{0}} \\ 0 & 0 & u_{0} & 0 & 0 \\ 0 & 0 & 0 & u_{0} & 0 \\ 0 & \kappa p_{0} & 0 & 0 & u_{0}\end{array}\right), \underline{A}_{2}=\left(\begin{array}{ccccc}v_{0} & 0 & \rho_{0} & 0 & 0 \\ 0 & v_{0} & 0 & 0 & 0 \\ 0 & 0 & v_{0} & 0 & \frac{1}{\rho_{0}} \\ 0 & 0 & 0 & v_{0} & 0 \\ 0 & 0 & \kappa p_{0} & 0 & v_{0}\end{array}\right), \underline{A}_{3}=\left(\begin{array}{ccccc}w_{0} & 0 & 0 & \rho_{0} & 0 \\ 0 & w_{0} & 0 & 0 & 0 \\ 0 & 0 & w_{0} & 0 & 0 \\ 0 & 0 & 0 & w_{0} & \frac{1}{\rho_{0}} \\ 0 & 0 & 0 & \kappa p_{0} & w_{0}\end{array}\right)$,

where $U_{0}:=\left(\rho_{0}, u_{0}, v_{0}, w_{0}, p_{0}\right)^{T}$ is the background flow. As an example, a planar wave is initialized such, that it contains only fluctuations in the right moving characteristic wave with the Eigenvalue $u_{0}+c_{0}$

$$
U=R W
$$


with $W=\hat{W} \sin (\vec{k} \cdot \vec{x})$ and the Eigenvector matrix

$$
R=\left(\begin{array}{ccccc}
n_{1} & n_{2} & n_{3} & \frac{\rho_{0}}{2 c_{0}} & \frac{\rho_{0}}{2 c_{0}} \\
0 & -n_{3} & n_{2} & \frac{n_{1}}{2} & -\frac{n_{1}}{2} \\
n_{3} & 0 & -n_{1} & \frac{n_{2}}{2} & -\frac{n_{2}}{2} \\
-n_{2} & n_{1} & 0 & \frac{n_{3}}{2} & -\frac{n_{3}}{2} \\
0 & 0 & 0 & \frac{\rho_{0}}{2 c_{0}} & \frac{\rho_{0}}{2 c_{0}}
\end{array}\right),
$$

with $c_{0}=\sqrt{\kappa \frac{p_{0}}{\rho_{0}}}$. We choose the perturbation of the characteristic variable vector $\hat{W}=(0.0,0.0,0.0,0.001,0.0)^{T}$, the normal vector of the wave $\vec{n}=(1.0,0.0,0.0)^{T}$, the wave number vector $\vec{k}=(\pi, 0.0,0.0)^{T}$ and the background flow

$U_{0}=\left(1.0,0.0,0.0,0.0, \frac{1}{\kappa}\right)^{T}$ with $\kappa=1.4$, resulting in $c_{0}=1.0$. The computational domain $\Omega:=[0.0 ; 2.0]^{3}$ is split into 8 regular subdomains $\Omega_{i}=\vec{x}_{i}+[0.0 ; 1.0]^{3}$, $i=1, \ldots, 8$ with

$$
\begin{aligned}
& \vec{x}_{1}:=(0.0,0.0,0.0)^{T}, \vec{x}_{2}:=(1.0,0.0,0.0)^{T}, \vec{x}_{3}:=(0.0,1.0,0.0)^{T}, \\
& \vec{x}_{4}:=(0.0,0.0,1.0)^{T}, \vec{x}_{5}:=(1.0,1.0,0.0)^{T}, \vec{x}_{6}:=(0.0,1.0,1.0)^{T}, \\
& \vec{x}_{7}:=(1.0,0.0,1.0)^{T}, \vec{x}_{8}:=(1.0,1.0,1.0)^{T} .
\end{aligned}
$$

For our $h$-refinement tests we introduce the parameter $n \geq 1$. For a given $n$, we first split every sub domain $\Omega_{i}$ into $n^{3}$ regular hexahedral elements. To generate the hybrid mesh, we furthermore split the hexahedra in the domain $i=1$ into tetrahedra, in the domains $i=2,3,4$ into prisms and in the domain $i=8$ into pyramids. We illustrate the different hexahedra splittings in figure 4 (please note that the front pyramid is blanked for better visualization purpose). For $n=1$ the hybrid prototype mesh consists of 21 grid cells.

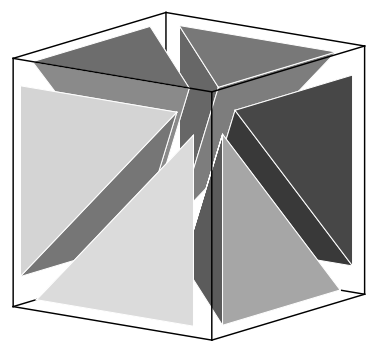

(a) 6 tetrahedra

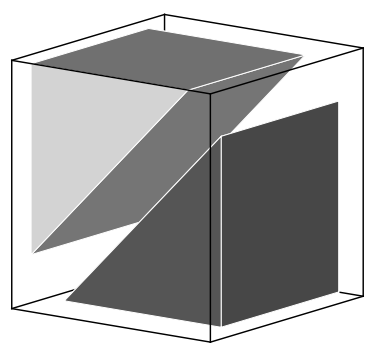

(b) 2 pentahedra

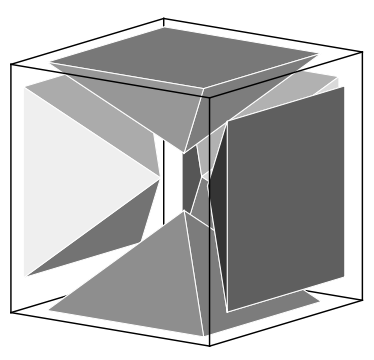

(c) 6 pyramids

Fig. 4. Visualization of the different hybrid meshes.

In table 4 the experimental order of convergence for this test case is plotted for $p=3$ and $p=4$. These results suggest that the order of the STE-DG discretization is $p+1$ in space and time. As expected, for the linear problem the results did not 


\begin{tabular}{cc|crr|rrr}
$\mathbf{n}$ & Nb cells & Nb DOF & $\boldsymbol{L}_{\mathbf{2}}\left(\boldsymbol{p}^{\prime}\right)$ & $\boldsymbol{E} \boldsymbol{O} \boldsymbol{C}$ & $\mathbf{N b} \mathbf{D O F}$ & $\boldsymbol{L}_{\mathbf{2}}(\boldsymbol{\rho} \boldsymbol{e})$ & $\boldsymbol{E O C}$ \\
\hline & & $\boldsymbol{p}=\mathbf{3}$ & & & $\boldsymbol{p}=\mathbf{4}$ & & \\
\hline 1 & 21 & 420 & $5,03 E-5$ & - & 9.408 & $3,51 E-6$ & - \\
2 & 168 & 3360 & $2,21 E-6$ & 4,5 & 75.264 & $1,22 E-7$ & 4,8 \\
3 & 567 & 11.340 & $4,22 E-7$ & 4,1 & 19.845 & $1,68 E-8$ & 4,9 \\
4 & 1344 & 26.880 & $1,22 E-7$ & 4,1 & 47.040 & $4,06 E-9$ & 4,9 \\
\hline
\end{tabular}

Experimental order of convergence for $p=3$ and $p=4$.

change when we increased the interpolation order $\widetilde{p}$ or when we changed the grid points via the parameters $\pi$. To further investigate the behavior of the discretization for different polynomial approximations, five configurations were tested. In the first configuration a fixed grid with $2^{3}$ hexahedral grid cells was used. We plot in figure 5 the $L_{2}$ error norm of the pressure $p^{\prime}$ for polynomial order $p=1$ up to $p=8$ with $t_{\text {end }}=20.0$. For the next configurations the hexahedral base grid was further split into tetrahedra, prisms or pyramids, according to figure 4, resulting in 48, 16 and 48 grid cells, respectively. In the last configuration the hybrid grid with $n=1$ was used, resulting in 21 grid cells. Please note that for the first four configurations the time steps do not differ over the computational domain, thus the local time stepping STE-DG scheme reduces to a global time stepping scheme. But for configuration five due to the different grid cell types and their different in-spheres, the scheme runs in local time stepping modus. It is interesting to compare for this test case the

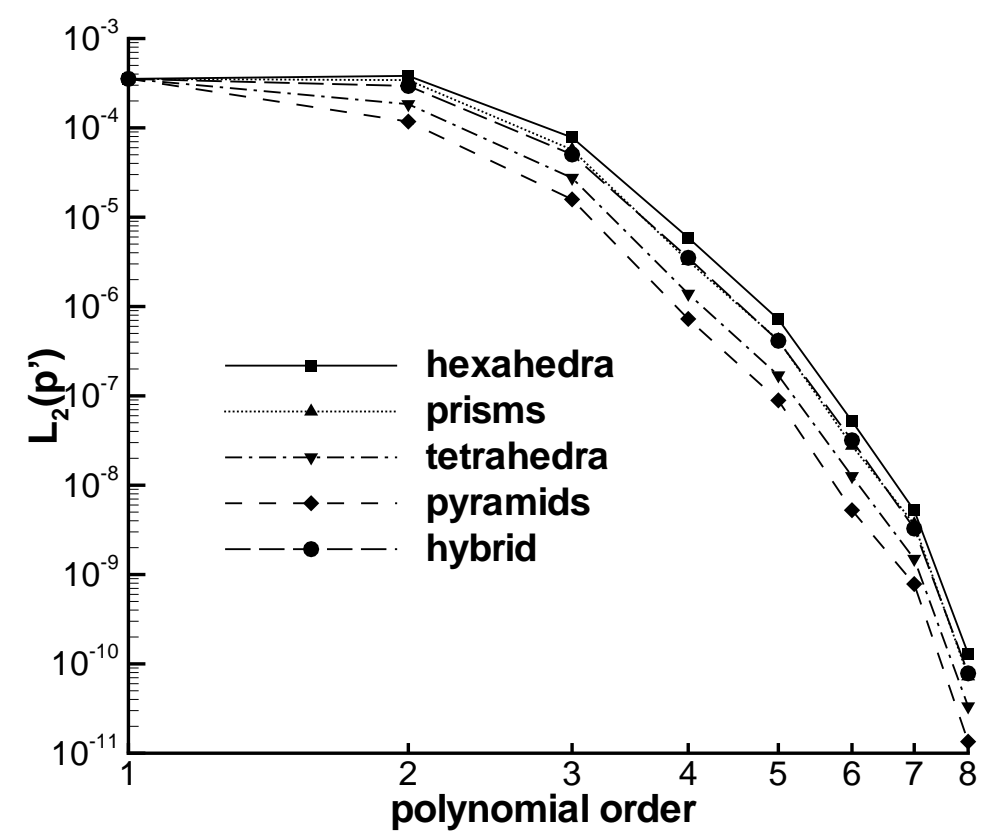

Fig. 5. Double logarithmic plot of $L_{2}$ error versus the polynomial order for different element types and grids. 
performances of the different grid cells. First of all comparing the number of grid cells in the different configurations and thus the number of DOF, figure 5 shows that the error norms do not differ much, thus uncovering a superior approximation behavior of the hexahedral grid cells compared to the other types. Furthermore if we compare the CPU time for the whole calculation, the hexahedral discretization succeeds again, as they allow larger time steps, resulting in the following ranking of this performance test: hexahedra (rel. CPU time $t=1$ ), prisms (rel. CPU time $t \approx 4$ ), tetrahedra (rel. CPU time $t \approx 10$ ) and pyramids (rel. CPU time $t \approx 20$ ). Several investigations indicate that this trends even hold true for non-linear problems, especially for the Navier-Stokes equations.

\subsection{The Euler equations}

In the following test, the influence of the recursion parameter $\pi=\left(\pi_{3 D}, \pi_{2 D}\right)$ and the influence of different interpolation orders is investigated. Based on the results from the linear test case, we consider in this subsection the non-linear Euler equations

$$
U_{t}+\vec{\nabla} \cdot \vec{F}(U)=0
$$

with the vector of the conservative variables $U=\left(\rho, \rho v_{1}, \rho v_{2}, \rho v_{3}, \rho e\right)^{T}$ and the Euler fluxes $\vec{F}:=\left(F_{1}, F_{2}, F_{3}\right)^{T}$ :

$$
F_{l}(U)=\left(\begin{array}{c}
\rho v_{l} \\
\rho v_{1} v_{l}+\delta_{1 l} p \\
\rho v_{2} v_{l}+\delta_{2 l} p \\
\rho v_{3} v_{l}+\delta_{3 l} p \\
\rho e v_{l}+p v_{l}
\end{array}\right), l=1,2,3 .
$$

Here, we use the usual nomination of the physical quantities: $\rho, \vec{v}=\left(v_{1}, v_{2}, v_{3}\right)^{T}$, $p$, and $e$ denote the density, the velocity vector, the pressure, and the specific total energy, respectively. Here the adiabatic exponent $\kappa=\frac{c_{p}}{c_{v}}$ with the specific heats $c_{p}, c_{v}$ depend on the fluid, and are supposed to be constant for this test. The system is closed with the equation of state of a perfect gas:

$$
p=\rho R T=(\kappa-1) \rho\left(e-\frac{1}{2} \vec{v} \cdot \vec{v}\right), \quad \text { and } \quad e=\frac{1}{2} \vec{v} \cdot \vec{v}+c_{v} T
$$

with the specific gas constant $R=c_{p}-c_{v}$. The considered test case is a three dimensional variation of the isentropic vortex convection problem of $\mathrm{Hu}$ and $\mathrm{Shu}$ 


$$
\begin{aligned}
& \vec{r}(\vec{x}, t)=\vec{r}_{\text {vortex }} \times\left(\vec{x}-\vec{x}_{0}-\vec{v}_{0} \cdot t\right), \\
& \delta v=\frac{v_{\text {max }}}{2 \pi} \exp \left(\frac{1-\left(\frac{|\vec{r}|}{r_{0}}\right)^{2}}{2}\right), \\
& \vec{v}(\vec{x}, t)=\vec{v}_{0}+\delta v \cdot \vec{r}, \\
& \frac{T}{T_{0}}=1-\frac{\kappa-1}{2}\left(\frac{\delta v}{c_{o}}\right)^{2}, \\
& \rho(\vec{x}, t)=\rho_{0}\left(\frac{T}{T_{0}}\right)^{\frac{1}{\kappa-1}}, \\
& p(\vec{x}, t)=p_{0}\left(\frac{T}{T_{0}}\right)^{\frac{\kappa}{\kappa-1}} .
\end{aligned}
$$

If we choose the rotational axis of the vortex $\vec{r}_{\text {vortex }}=(0 ., 0 ., 1 .)^{T}$ and $\rho_{0}=p_{0}=$ $R=1$, then the standard two dimensional problem is recovered. For our test problem we chose the background flow $\left(\rho_{0}, \vec{v}_{0}^{T}, p_{0}\right)=\left(1 ., 1 ., 1 ., 1 ., \frac{1}{\kappa}\right), \kappa=1.4$, the rotational axis of the vortex $\vec{r}_{\text {vortex }}=(1 .,-0.5,1 .)^{T}$, the initial center of the vortex $\vec{x}_{0}=(0.5,0.5,0.5)^{T}$, the amplitude of the vortex $v_{\max }=0.1$, the halfwidth of the vortex $r_{0}=1.0$ and the endtime of the simulation $t_{\text {end }}=4.0$. The computational domain $\Omega:=[0.0,5.0]^{3}$ with exact boundary conditions prescribed. The solution to this problem at time $t=2.0$ with $6^{3} p=5$ hexahedra is shown in figure 6 . The results of tests with $p=6$ trial functions with different parameters $\pi$ and/or different interpolation orders $\widetilde{p}$ are listed in tables 5 - 8 .

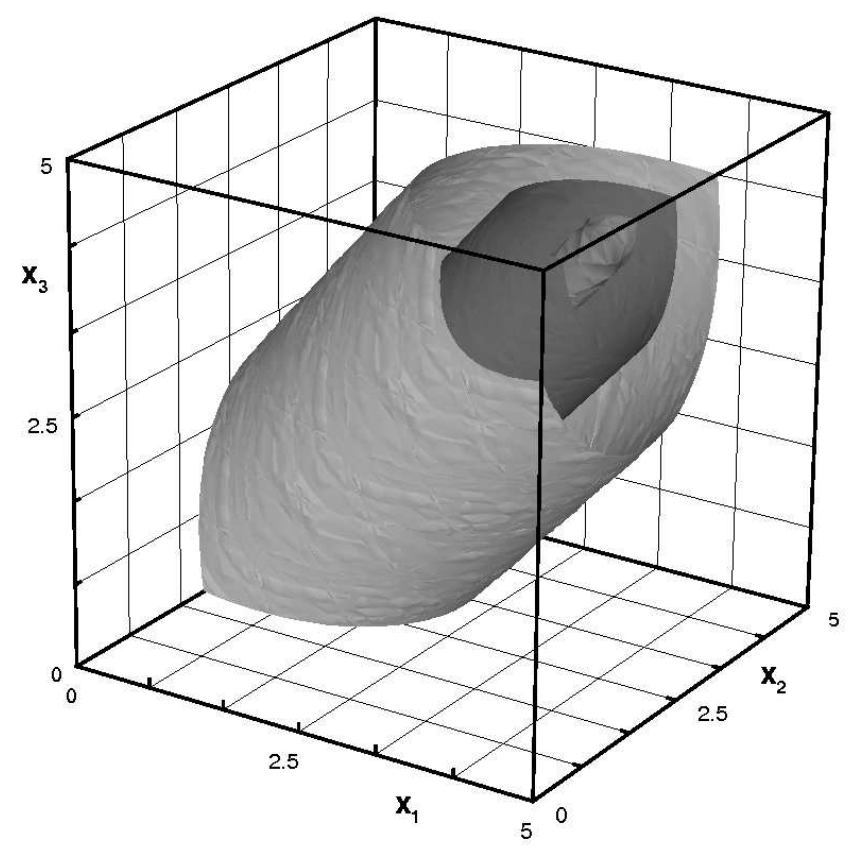

Fig. 6. 3D isentropic vortex. Isosurfaces of density ( $\rho=0.99977,99989,99998)$. 


\begin{tabular}{cc|cc} 
Interpolation order $(\widetilde{p})$ and $\pi$ & Nb Int points & $\boldsymbol{L}_{\mathbf{2}}(\boldsymbol{\rho})$ & CPU time/EU \\
\hline$\widetilde{p}=6, \pi=(0,0)$ & 117 & $1,9654 E-05$ & $100 \%$ \\
$\widetilde{p}=6, \pi=(1,0)$ & 124 & $1,7455 E-05$ & $107 \%$ \\
$\widetilde{p}=6, \pi=(0,1)$ & 147 & $1,8112 E-05$ & $120 \%$ \\
$\widetilde{p}=6, \pi=(1,1)$ & 154 & $1,6055 E-05$ & $121 \%$ \\
$\widetilde{p}=6, \pi=(2,0)$ & 136 & $1,7399 E-05$ & $110 \%$ \\
$\widetilde{p}=6, \pi=(2,1)$ & 166 & $1,5832 E-05$ & $125 \%$ \\
\hline$\widetilde{p}=7, \pi=(0,0)$ & 160 & $1,7586 E-05$ & $127 \%$ \\
$\widetilde{p}=8, \pi=(0,0)$ & 214 & $1,6336 E-05$ & $154 \%$ \\
$\widetilde{p}=7, \pi=(4,2)$ & 512 & $1,4770 E-05$ & $255 \%$ \\
\hline Gauss Legendre points & 637 & $1,4665 E-05$ & $403 \%$ \\
\hline
\end{tabular}

Table 5

Results for different types of integration points for $p=6$ hexahedra. The domain $\Omega$ is subdivided into 8 hexahedra.

\begin{tabular}{cc|cc} 
Interpolation order $(\widetilde{p})$ and $\pi$ & Nb Int points & $\boldsymbol{L}_{\mathbf{2}}(\boldsymbol{\rho})$ & CPU time/EU \\
\hline$\widetilde{p}=6, \pi=(0,0)$ & 98 & $2,8744 E-04$ & $100 \%$ \\
$\widetilde{p}=6, \pi=(1,0)$ & 106 & $2,8256 E-04$ & $109 \%$ \\
$\widetilde{p}=6, \pi=(0,1)$ & 103 & $1,7078 E-04$ & $107 \%$ \\
$\widetilde{p}=6, \pi=(1,1)$ & 111 & $1,6332 E-04$ & $110 \%$ \\
\hline$\widetilde{p}=7, \pi=(0,0)$ & 138 & $2,7298 E-04$ & $127 \%$ \\
$\widetilde{p}=8, \pi=(0,0)$ & 187 & $1,5537 E-04$ & $181 \%$ \\
$\widetilde{p}=7, \pi=(1,1)$ & 159 & $1,0978 E-04$ & $136 \%$ \\
\hline Gauss Jacobi points & 588 & $9,8771 E-05$ & $425 \%$ \\
\hline
\end{tabular}

Table 6

Results for different types of integration points for $p=6$ pyramids. The domain $\Omega$ is subdivided into 6 pyramids.

The general observation is, that if we increase the number of interpolation points, then the error norm decreases and the CPU time increases. We also compared the nodal integration to the standard Gaussian integration, where we chose $7^{3}=343$ tensor product Jacobi Gauss points for the volume integrals and $7^{2}=49$ tensor product Jacobi Gauss points for each of the surface integrals. Although the results with standard Gauss cubature are slightly more accurate, comparing CPU times clearly confirms that the nodal type integration is more efficient. 


\begin{tabular}{cc|cc} 
Interpolation order $(\widetilde{p})$ and $\pi$ & Nb Int points & $\boldsymbol{L}_{\mathbf{2}}(\boldsymbol{\rho})$ & CPU time/EU \\
\hline$\widetilde{p}=6, \pi=(0,0)$ & 101 & $1,4853 E-05$ & $100 \%$ \\
$\widetilde{p}=6, \pi=(1,0)$ & 110 & $1,4235 E-05$ & $109 \%$ \\
$\widetilde{p}=6, \pi=(0,1)$ & 116 & $1,2260 E-05$ & $114 \%$ \\
$\widetilde{p}=6, \pi=(1,1)$ & 125 & $1,2250 E-05$ & $118 \%$ \\
\hline$\widetilde{p}=7, \pi=(0,0)$ & 141 & $1,4210 E-05$ & $127 \%$ \\
$\widetilde{p}=8, \pi=(0,0)$ & 188 & $1,2925 E-05$ & $154 \%$ \\
$\widetilde{p}=7, \pi=(0,1)$ & 165 & $1,1562 E-05$ & $141 \%$ \\
\hline Gauss Jacobi points & 588 & $1,1006 E-05$ & $424 \%$ \\
\hline
\end{tabular}

Table 7

Results for different types of integration points for $p=6$ prisms. The domain $\Omega$ is subdivided into 8 hexahedra which are further subdivided into 2 prisms, yielding 16 grid cells.

\begin{tabular}{cc|cc} 
Interpolation order $(\widetilde{p})$ and $\pi$ & Nb Int points & $\boldsymbol{L}_{\mathbf{2}}(\boldsymbol{\rho})$ & CPU time/EU \\
\hline$\widetilde{p}=6, \pi=(0,0)$ & 84 & $1,414 E-04$ & $100 \%$ \\
\hline$\widetilde{p}=7, \pi=(0,0)$ & 120 & $1,4386 E-04$ & $113 \%$ \\
$\widetilde{p}=8, \pi=(0,0)$ & 165 & $1,3945 E-04$ & $135 \%$ \\
\hline Gauss Jacobi points & 539 & $1,3790 E-04$ & $399 \%$ \\
\hline
\end{tabular}

Table 8

Results for different types of integration points for $p=6$ tetrahedra. The domain $\Omega$ is subdivided into 6 tetrahedra.

\subsection{Compressible Navier-Stokes equations}

The three dimensional unsteady compressible Navier-Stokes equations with a source term reads as

$$
U_{t}+\vec{\nabla} \cdot \vec{F}(U)-\vec{\nabla} \cdot \vec{F}^{v}(U, \vec{\nabla} U)=S,
$$

with the vector of the conservative variables $U$, the non-linear Euler fluxes $\vec{F}:=$ $\left(F_{1}, F_{2}, F_{3}\right)^{T}$ and the diffusion fluxes $\vec{F}^{v}:=\left(F_{1}^{v}, F_{2}^{v}, F_{3}^{v}\right)^{T}$ :

$$
F_{l}^{v}(U, \vec{\nabla} U)=\left(\begin{array}{c}
0 \\
\tau_{1 l} \\
\tau_{2 l} \\
\tau_{3 l} \\
\tau_{l j} v_{j}-q_{l}
\end{array}\right), l=1,2,3
$$


The viscous stress tensor is given by

$$
\underline{\tau}:=\mu\left(\vec{\nabla} \vec{v}+(\vec{\nabla} \vec{v})^{T}-\frac{2}{3}(\vec{\nabla} \cdot \vec{v}) \underline{I}\right),
$$

and the heat flux by $\vec{q}=\left(q_{1}, q_{2}, q_{3}\right)^{T}$ with

$$
\vec{q}:=-k \vec{\nabla} T, \quad \text { with } \quad k=\frac{c_{p} \mu}{P r},
$$

Here, the viscosity coefficient $\mu$ and the Prandtl number $\operatorname{Pr}$ depend on the fluid, and are supposed to be constant for this test. If we choose

$$
S=\alpha\left(\begin{array}{c}
\cos (\beta)(d k-\omega) \\
\cos (\beta) A+\sin (2 \beta) \alpha k(\kappa-1) \\
\cos (\beta) A+\sin (2 \beta) \alpha k(\kappa-1) \\
\cos (\beta) A+\sin (2 \beta) \alpha k(\kappa-1) \\
\cos (\beta) B+\sin (2 \beta) \alpha(d k \kappa-\omega)+\sin (\beta)\left(\frac{d k^{2} \mu \kappa}{P r}\right)
\end{array}\right)
$$

with $\beta:=k\left(x_{1}+x_{2}+x_{3}\right)-\omega t, A=-\omega+\frac{k}{d-1}\left((-1)^{d-1}+\kappa(2 d-1)\right)$ and $B=\frac{1}{2}\left(\left(d^{2}+\kappa(6+3 d)\right) k-8 \omega\right)$, the analytical solution to (36)+(40) is given by

$$
U=\left(\sin (\beta) \alpha+2, \sin (\beta) \alpha+2, \sin (\beta) \alpha+2, \sin (\beta) \alpha+2,(\sin (\beta) \alpha+2)^{2}\right)^{T} .
$$

For our test we choose the coefficients $\kappa=1.4, \operatorname{Pr}=0.72, \mu=0.0001, R=$ 287.14 and $\alpha=0.5, \omega=10.0, k=\pi$ with the dimension of the problem $d=3$. We solve this problem with the recently developed modal STE-DG scheme for compressible Navier-Stokes equations [15], with the above presented nodal modifications. The main building block of this discretization is a new weak formulation, where integration by parts is used twice, circumventing the need for resorting to a mixed first order system and thus circumventing the need for additional auxiliary variables. For the numerical fluxes we choose approximate Riemann solvers for both, the hyperbolic part and the parabolic part. For the approximation of the Euler flux we choose the HLLC flux [37] and for the approximation of the viscous fluxes the recently developed dGRP flux [14], [15], [30], which can be interpreted as a natural extension of the classic interior penalty flux [33] for the Laplace equation to the viscous terms of the compressible Navier-Stokes equations. The results of a convergence test with the hybrid grids from example 4.1 are listed in table 9 for $p=4$ and $p=5$ with $\pi=(0,0)$, where we used $t_{\text {end }}=1.0$ and periodic boundary conditions. The results indicate that the optimal order of convergence $E O C=p+1$, for $p$ odd and even, is achieved.

We list the average CPU time per element update and per degree of freedom (CPU/EU/DOF) for the 3D compressible Navier-Stokes equations with $p=6$ Ansatz functions ( 84 


\begin{tabular}{cc|ccc|ccr}
$\mathbf{n}$ & Nb cells & Nb DOF & $\boldsymbol{L}_{\mathbf{2}}(\boldsymbol{\rho} \boldsymbol{e})$ & $\boldsymbol{E} \boldsymbol{O} \boldsymbol{C}$ & $\mathbf{N b}$ DOF & $\boldsymbol{L}_{\mathbf{2}}(\boldsymbol{\rho} \boldsymbol{e})$ & $\boldsymbol{E} \boldsymbol{O C}$ \\
\hline & & $\boldsymbol{p}=\mathbf{4}$ & & & $\boldsymbol{p}=\mathbf{5}$ & & \\
\hline 2 & 168 & 5.880 & $6,13 E-3$ & - & 9.408 & $3,80 E-3$ & - \\
4 & 1344 & 47.040 & $1,91 E-4$ & 5,0 & 75.264 & $9,36 E-5$ & 5,3 \\
8 & 10752 & 376.320 & $4,32 E-6$ & 5,5 & 602.112 & $1,54 E-6$ & 5,9 \\
16 & 86016 & 3.010 .560 & $1,22 E-7$ & 5,1 & 4.816 .896 & $2,38 E-8$ & 6,0 \\
\hline
\end{tabular}

Experimental order of convergence for $p=4$ and $p=5$ with $\pi=(0,0)$ and $t_{\text {end }}=1.0$.

DOF/Element) in table 10. Based on the investigations in subsection 4.2, we chose for every grid cell type the most efficient combination (in terms of accuracy versus cpu time) of the parameters $\pi$ and the interpolation order $\widetilde{p}$. All CPU times were measured on one processor of a Intel Xeon Dual Core CPU with $2.66 \mathrm{GHz}$. An equivalent measurement for a 6 th order compact finite difference scheme with 4 th order Runge-Kutta time integration, [2], on the same CPU yields $\sim 56,0 \mu \mathrm{s}$.

\begin{tabular}{|c|c|c|}
\hline Interpolation order $(\widetilde{p})$ and $\pi$ & Element type & CPU time/EU/DOF $[\mu$ s] \\
\hline$\widetilde{p}=6, \pi=(1,1)$ & hexahedron & 39,9 \\
\hline$\widetilde{p}=7, \pi=(1,1)$ & pyramid & 43,1 \\
\hline$\widetilde{p}=6, \pi=(0,1)$ & prism & 31,5 \\
\hline$\widetilde{p}=6, \pi=(0,0)$ & tetrahedron & 27,7 \\
\hline
\end{tabular}

Table $\overline{10}$

CPU times for the 3D compressible Navier-Stokes equations with $(p=6)$ STE-DG discretization ( $7 t h$ order in space and time).

\subsubsection{Polygonal meshes}

In this section preliminary results for a DG discretization with polygonal meshes are shown. We propose to apply the recursion based algorithm to define efficient sets of interpolation points for polygonal grid cells. Numerical investigations indicate that for a general grid cell the shape dependent parameter $p^{*}$, which is the maximal possible interpolation order with surface points only, has the value 'number of sides minus one', which we choose for all grid cell types (2D and 3D) discussed in this work. Starting from a triangle mesh the corresponding dual mesh is constructed and used as polygonal mesh, figure $7 \mathrm{a}$. The primal triangle mesh is no longer needed as it is only used to construct the dual mesh. The resulting polygonal mesh contains elements with 4 sides up to element with 7 sides. For the distribution of the interpolation points two different strategies are used for an approximation with $p=3$. For the first strategy we directly use the recursion algorithm (14) with a fixed recursion parameter $\pi_{2 D}$ for all elements. If we choose $\pi_{2 D}=0$, test con- 


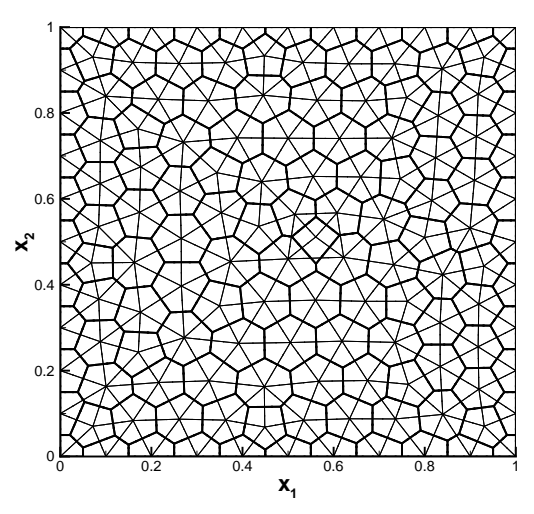

(a) primal and dual mesh

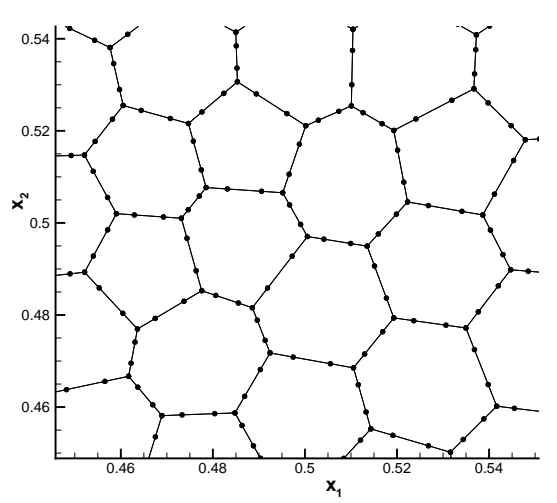

(b) configuration A

Fig. 7. Primal and dual mesh $(h=0.1)$ and detailed view of the interpolation grid ( $h=0.025)$ with $p=3\left(\pi_{2 D}=0\right)$ interpolation.

figuration A shown in figure 7b, the resulting interpolation grid is only distributed at grid cell boundaries, as all grid cells have at least 4 sides. Similar to the discussion above it is favorable for non-linear problems to use more interpolation points, i.e. increasing the recursion parameter $\pi_{2 D}$. In figure $8 \mathrm{a}$ the recursion parameter is set to $\pi_{2 D}=3$, test configuration $\mathrm{B}$, for all grid cells. In this extreme case where quadrilaterals (4 sides, 3 recursive defined interior point layers) and heptagons (7 sides, 0 recursive defined interior point layers) arise, the resulting point distribution is non-uniform and seems to be not well suited. To circumvent this, our second strategy is to fix the number of recursions for every grid cell type, thus introducing the recursion parameter $\pi_{2 D}$ independently for every grid cell type. In figure $8 \mathrm{~b}$ the interpolation grid for a fixed recursion number $r_{\max }=1$, test configuration $\mathrm{C}$, with a second order inner point distribution is shown, corresponding to the parameter $\pi_{2 D}=2$ for quadrilaterals and $\pi_{2 D}=5$ for heptagons. To validate this discretiza-

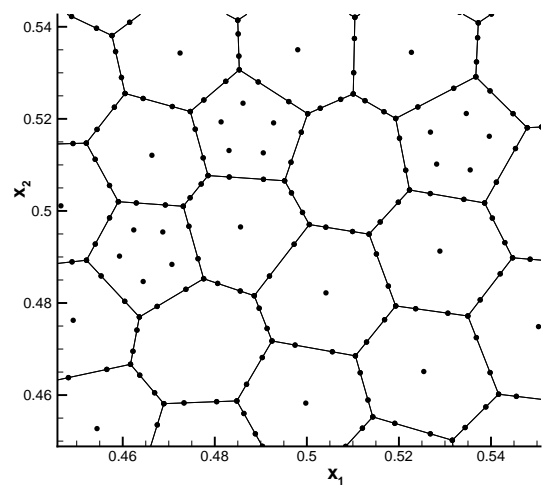

(a) configuration $\mathrm{B}$

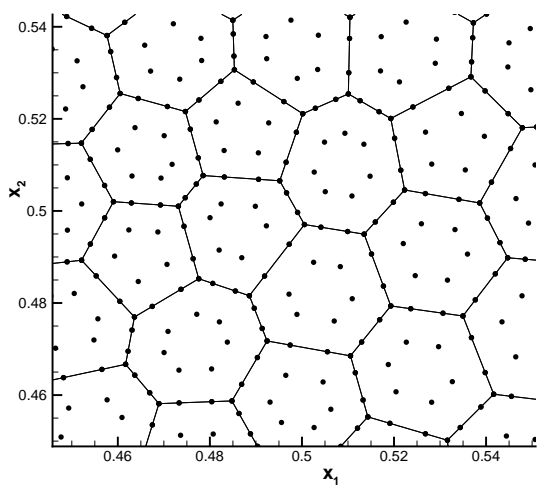

(b) configuration $\mathrm{C}$

Fig. 8. Detailed view of the interpolation grid $(h=0.025)$ for $p=3$ approximation with $\pi_{2 D}=3$ or $r_{\max }=1$.

tions the compressible Navier-Stokes equations with a source term are considered, where we used the reduced two dimensional version of the previous example with the same parameters, excepting the parameter $k$ which we changed from $\pi$ to $2 \pi$ 
and the dimension $\mathrm{d}$ from 3 to 2 . For the grid refinement, four different regular triangle grids with typical mesh size $h$ are constructed and then converted to polygonal meshes, similar to 7a. The pre-computation of the surface and volume integral matrices is done on sub triangles with standard Gaussian integration. In table 11 the results for configuration $\mathrm{A}$ and the results for the reference computation on the primal triangular grid with $t_{\text {end }}=0.5$ and exact boundary conditions are shown. We notice first that the expected order of convergence is achieved. Considering efficiency, the results on the primal mesh are more accurate, whereas the CPU time for configuration $\mathrm{A}$ is $t_{C P U}=378 \mathrm{~s}$ and the $\mathrm{CPU}$ time for the primal configuration is $t_{C P U}=594 \mathrm{~s}$. The reasons for the CPU time advantage is, that the resulting polygonal configuration has only about half the DOF and allows larger (explicit) time steps. To account for the non-linearity of the Navier-Stokes fluxes, computations

Table 11

\begin{tabular}{c|ccc|ccc}
$\mathbf{h}$ & $\mathbf{N b}$ cells & $\boldsymbol{L}_{\mathbf{2}}(\boldsymbol{\rho} \boldsymbol{e})$ & $\boldsymbol{E} \boldsymbol{O C}$ & $\mathbf{N b}$ cells & $\boldsymbol{L}_{\mathbf{2}}(\boldsymbol{\rho} \boldsymbol{e})$ & $\boldsymbol{E} \boldsymbol{O C}$ \\
\hline & \multicolumn{4}{|c|}{ triangular configuration } & \multicolumn{3}{c}{ configuration A } \\
\hline 0,2 & 62 & $2,44 E-3$ & - & 42 & $1,28 E-2$ & - \\
0,1 & 226 & $1,92 E-4$ & 3,7 & 134 & $1,31 E-3$ & 3,3 \\
0,05 & 896 & $1,07 E-5$ & 4,3 & 489 & $7,16 E-5$ & 4,2 \\
0,025 & 3595 & $6,42 E-7$ & 4,1 & 1878 & $4,77 E-6$ & 3,9 \\
\hline 11
\end{tabular}

Experimental order of convergence for $p=3$ (10 DOF per grid cell) for reference test on primal triangular mesh and for test configuration $\mathrm{A}$.

with configuration $\mathrm{B}$ and $\mathrm{C}$ are performed and corresponding results are listed in table 12. We notice that the accuracy of the solution is improved, approaching the quality of the primal configuration solution. As expected, the results of configuration $\mathrm{C}$ are more accurate compared to the results of configuration $\mathrm{B}$. Considering the efficiency of the computations, the CPU time for test B is $t_{C P U}=380 \mathrm{~s}$ and for test $\mathrm{C} t_{C P U}=398 \mathrm{~s}$ showing a large potential for DG discretizations on polygonal meshes compared to traditional triangular meshes. In future future works we

Table 12

\begin{tabular}{cc|cc|cc}
$\mathbf{h}$ & Nb cells & $\boldsymbol{L}_{\mathbf{2}}(\boldsymbol{\rho} \boldsymbol{e})$ & $\boldsymbol{E} \boldsymbol{O C}$ & $\boldsymbol{L}_{\mathbf{2}}(\boldsymbol{\rho} \boldsymbol{e})$ & $\boldsymbol{E} \boldsymbol{O C}$ \\
\hline & & \multicolumn{2}{|c|}{ configuration B } & \multicolumn{2}{|c}{ configuration C } \\
\hline 0,2 & 42 & $9,55 E-3$ & - & $5,17 E-3$ & - \\
0,1 & 134 & $7,22 E-4$ & 3,7 & $4,25 E-4$ & 3,6 \\
0,05 & 489 & $3,38 E-5$ & 4,4 & $2,64 E-5$ & 4,0 \\
0,025 & 1878 & $1,84 E-6$ & 4,2 & $1,64 E-6$ & 4,0 \\
\hline
\end{tabular}

Experimental order of convergence for $p=3$ (10 DOF per grid cell) for test configuration $\mathrm{B}$ and $\mathrm{C}$.

will investigate the influence of different node distribution strategies and recursion 
parameters for polygonal meshes and furthermore investigate the applicability of the recursion algorithm (16) with ' $p^{*}=$ number of sides minus 1' for polyhedral meshes in three dimensions.

\subsubsection{Boundary Layer Instability}

We consider in this example the evolution of a Tolmien-Schlichting wave in a subsonic compressible boundary layer. The computational domain $\Omega$ extends from $x_{1}=337.0$ to $x_{1}=890.0$ and $x_{2}=0.0$ to $x_{2}=22.35$. We choose subsonic inflow and outflow boundary conditions and at $x_{2}=0.0$ isothermal wall conditions with $T_{w}=296.0 \mathrm{~K}$. The initial solution of the computation is obtained from a similarity solution with Mach number $M_{\infty}=0.8$ and $T_{\infty}=280.0 \mathrm{~K}$. The Reynolds number $R e:=\frac{\rho_{\infty} v_{1} \delta_{1}}{\mu\left(T_{\infty}\right)}=1000$, based on the displacement thickness at the inflow $\delta_{1}$. Using $\delta_{1}$ as the reference length, we get $\delta_{1}=1.0$ at the inflow and the boundary layer thickness $\delta_{99}=2.95$ and $\delta_{99}=4.8$ at the inflow and outflow, respectively. The temperature dependence of viscosity $\mu$ is modeled using Sutherland's law

$$
\mu(T)=\mu\left(T_{\infty}\right) T^{3 / 2} \frac{1+T_{s}}{T+T_{s}}
$$

with $\mu\left(T_{\infty}\right)=1.73510^{-5} \frac{\mathrm{kg}}{\mathrm{ms}}$ and $T_{s}=110.4 \mathrm{~K}$.

The inflow at $x_{1}=337.0$ is superimposed with a forcing term, composed of the eigenfunction of the Tolmien-Schlichting wave with the fundamental frequency $\omega_{0}=0.0688$. For a detailed description of the similarity solution and the eigenfunction we refer to Babucke et al. [1]. The computational domain was subdivided in $48 \times 22$ regular quadrilaterals and discretized with $p=6\left(\pi_{2 D}=1\right)$ STEDG scheme, resulting in 29568 DOF. The endtime of the simulation was set to $\frac{t_{\text {end }}}{T_{0}}=37$, where $T_{0}=\frac{2 \pi}{\omega_{0}} \approx 92$, to ensure a periodic solution. To analyze our results we apply a discrete Fourier analysis using one period of the forcing frequency $T_{0}$ from $\frac{t}{T_{0}}=36$ to $\frac{t}{T_{0}}=37$. We plot the maximal amplitude of $v_{1}$ with respect to $x_{2}$ as a function of $x_{1}$ in figure 9. For comparison, corresponding results obtained with a 6th order compact finite difference code with $330 \times 150$ grid points and 4th order Runge Kutta time integration [1] are included, showing good agreement. We furthermore plot the amplification rate $\alpha_{i}$ of the velocity $v_{1}$ based on the maximal amplitude in figure 9. Again, the result is in good accordance to the reference result [1] and the predictions of linear stability theory.

\subsubsection{Flow past a Sphere at $R e=300$}

We consider in this example a sphere with radius $r=1$ centered at $\vec{x}_{0}=(0,0,0)^{T}$. We solve the 3D unsteady compressible Navier-Stokes equations with Mach number $M=0.3$ and Reynolds number $R e=300$ based on the diameter of the sphere. The computational domain extends from $x_{1}=-20.0$ to $x_{1}=100.0$ and $x_{2}, x_{3}= \pm 30.0$. The grid consists of $\approx 160.000$ tetrahedra, where the wake of the 

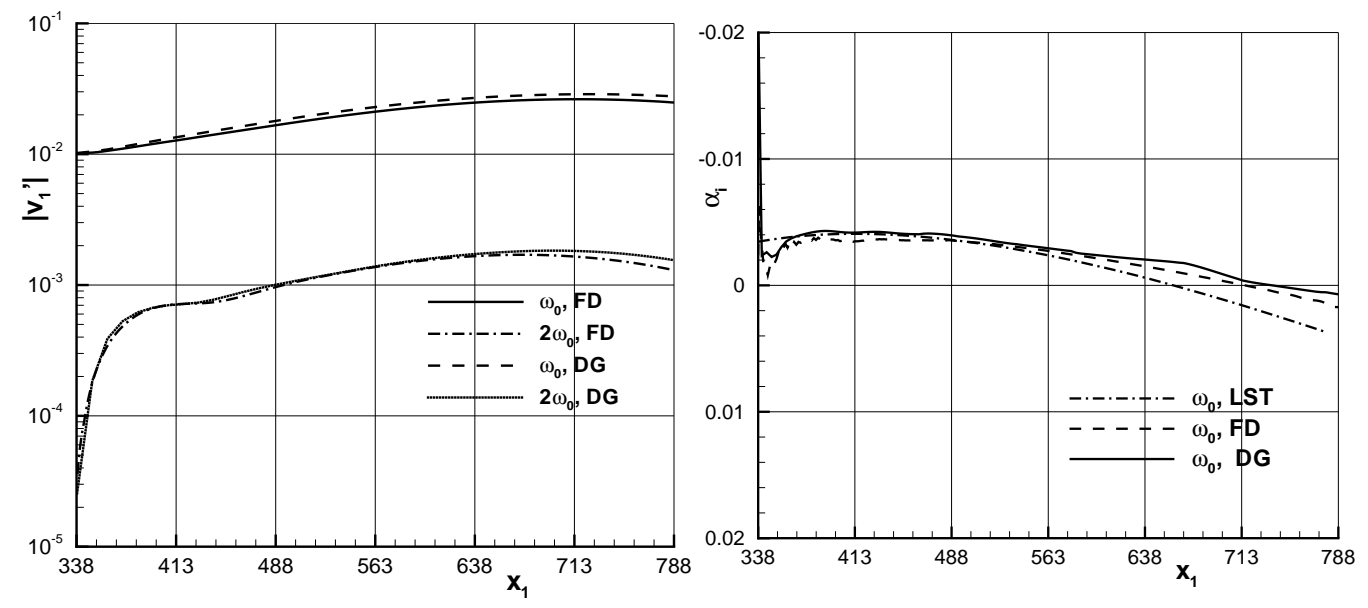

Fig. 9. Maximum amplitudes of $v_{1}$ (left). Amplification rate $\alpha_{i}$ of $u_{1}$ based on maximum amplitude (right).

sphere is resolved with $h \approx 0.4$. The surface of the sphere is discretized using triangles with $h \approx 0.1$. To capture the right geometry of the sphere, tetrahedra with curved boundary surfaces are used. We plot the cut of the grid on a cut plane with $\vec{n}_{\text {plane }}=(0,1,0)^{T}$ in figure 10 . For the calculation the $p=3$ STE-DG scheme was

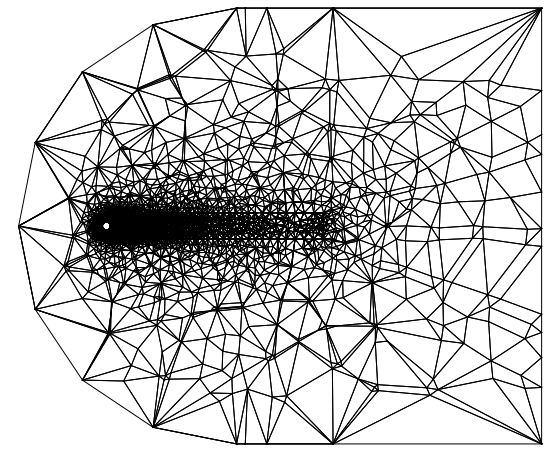

(a) total grid

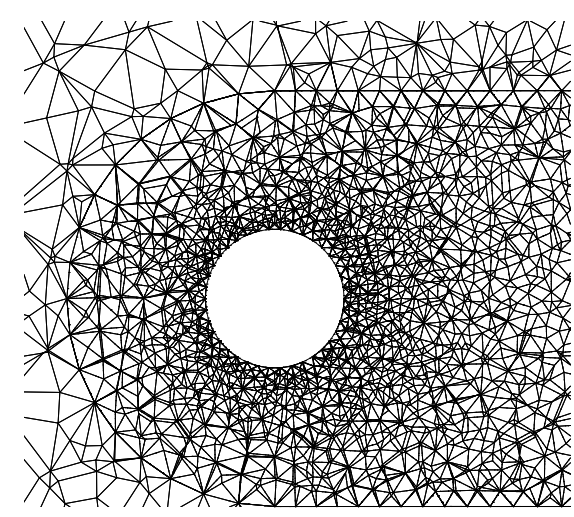

(b) zoomed grid

Fig. 10. Visualization of the grid for the sphere example.

used, resulting in $\approx 3.000 .000$ DOF. A contour plot of the velocity magnitude, figure 11, shows that the boundary layer is resolved within 1-2 tetrahedral elements. In figure 12 the structure of the vortices are shown using the $\lambda_{2}$ vortex detection criterium. We list in table 13 the resulting force coefficients, the corresponding oscillating amplitudes and the Strouhal number Str. For comparison results from Tomboulides [36] and Johnson and Patel [27], obtained within an incompressible simulation, are listed as well. In figure 13 we plot the drag coefficient $C_{d}$ and the lateral force coefficient $C_{l}$ versus time $t$. 


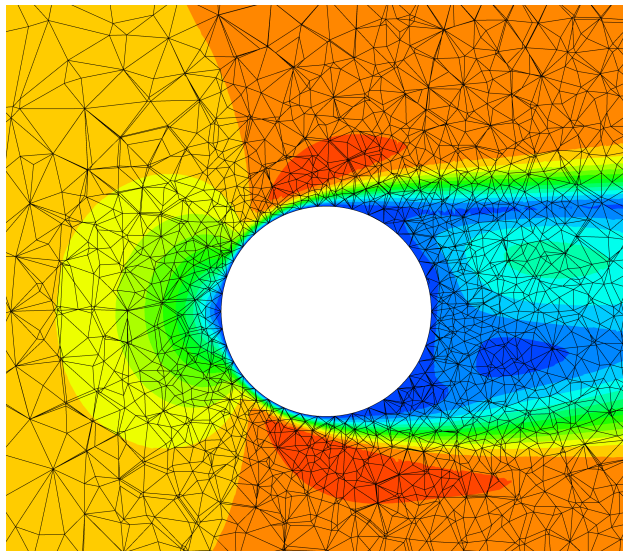

(a) velocity magnitude

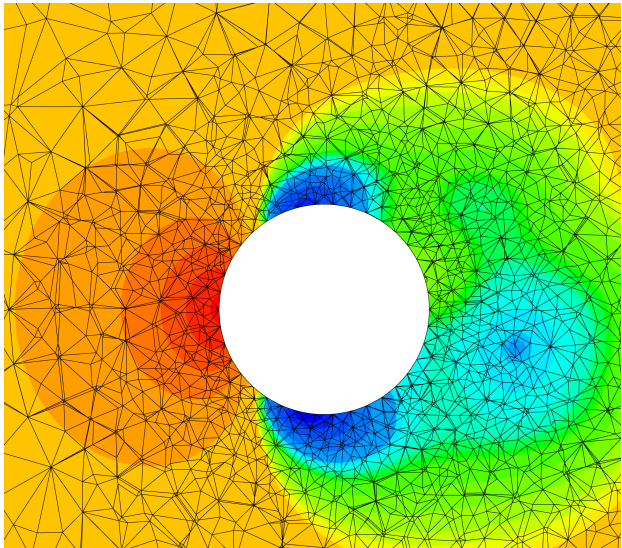

(b) pressure

Fig. 11. Contour plot of the instantaneous velocity magnitude $|\vec{v}|=0.0 \ldots 0.3478$ and pressure $p=0.688 \ldots 0.762$.

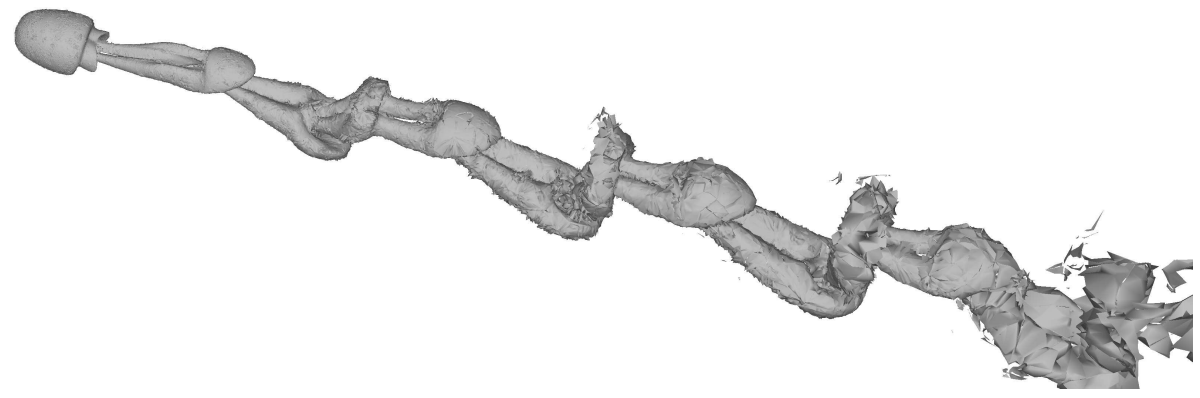

Fig. 12. Isometric view of $\lambda_{2}$ isosurface.

\begin{tabular}{c|ccccc} 
& $C_{d}$ & $\Delta C_{d}$ & $C_{l}$ & $\Delta C_{l}$ & $S t r$ \\
\hline & 0.673 & 0.0031 & -0.065 & 0.015 & 0.135 \\
Tomboulides [36] & 0.671 & 0.0028 & - & - & 0.136 \\
Tohnson\&Patel [27] & 0.656 & 0.0035 & -0.069 & 0.016 & 0.137 \\
\hline
\end{tabular}

Table 13

Force coefficients and Strouhal number. 


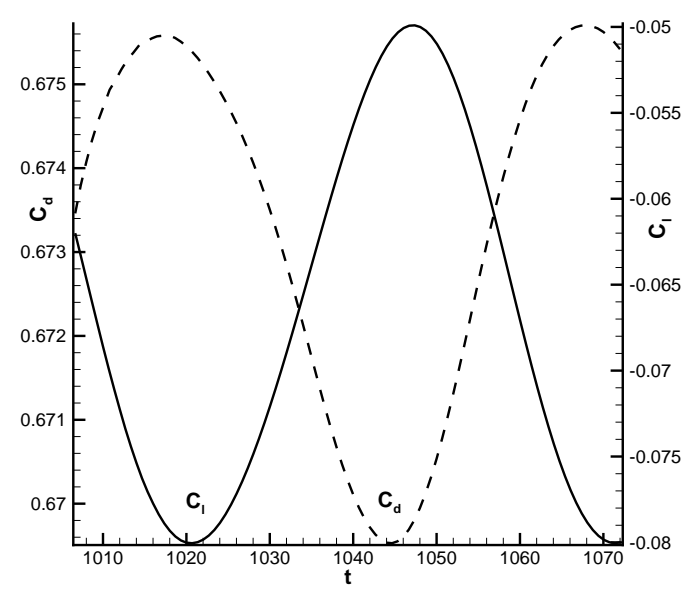

Fig. 13. Drag and lateral force coefficient. 


\section{Conclusion}

Part one of this paper deals with a framework for efficient polynomial interpolation on polymorphic grid cells, i.e. the definition of a nodal interpolation basis. In our framework, for non simplex grid cells the number of nodal basis functions is higher than the number of modal basis functions. We showed that one way to get a reasonable Vandermonde matrix is to use the singular value decomposition framework to build a least squares inverse. The properties of these Vandermonde matrices (and the corresponding interpolation) solely depend on the position of the interpolation points. We consider in this paper only interpolation points with a symmetric distribution, points which support an interpolation of order $p$ in the volume of the grid cell and simultaneously an interpolation of the same order on each of the faces of the grid cell. We therefore introduced a simple construction guideline, which is based on a recursive algorithm starting from a given surface points distribution. Using a set of $1 \mathrm{D}$ points, we can successive define points for $2 \mathrm{D}$ faces, and consequently define points for 3D volumes.

In the second part of the paper we introduced a novel integration framework for modal discontinuous Galerkin schemes, which could be easily implemented in an existing Gauss integration based modal DG code. Borrowing from nodal methods a mixed modal-nodal DG scheme was constructed. As an example the nodal based integration was combined with the recently developed space-time expansion discontinuous Galerkin scheme yielding an efficient high order discretization on arbitrary unstructured grids for unsteady flow problems.

\section{Acknowledgments}

This project was supported by the Deutsche Forschungsgemeinschaft (DFG). The authors would further like to thank Andreas Babucke for providing the initial condition and the eigensolutions for the Tolmien-Schlichting wave example. 


\section{References}

[1] Babucke, A., Kloker, M. J., And Rist, U. Finite differences on non-uniform grids. submitted to J. Comput. Phys.

[2] Babucke, A., Kloker, M. J., AND Rist, U. DNS of a plane mixing layer for the investigation of sound generation mechanisms. to appear in Computers and Fluids (2007). DOI:10.1016/j.compfluid.2007.02.002.

[3] BASSI, F., AND REBAY, S. A high-order accurate discontinuous finite element method for the numerical solution of the compressible Navier-Stokes equations. J. Comput. Phys. 131 (1997), 267-279.

[4] BASSi, F., AND Rebay, S. A high-order discontinuous Galerkin finite element method solution of the 2d euler equations. J. Comput. Phys. 138 (1997), 251-285.

[5] Bassi, F., AND Rebay, S. Numerical evaluation of two discontinuous Galerkin methods for the compressible Navier-Stokes equations. International Journal for Numerical Methods in Fluids 40 (2002), 197-207.

[6] Cheng, Y., AND Shu, C.-W. A discontinuous Galerkin finite element method for time dependent partial differential equations with higher order derivatives. Math. Comp (2007). to appear.

[7] Cockburn, B., Hou, S., And Shu, C. W. The Runge-Kutta local projection discontinuous Galerkin finite element method for conservation laws IV: The multidimensional case. Math. Comput. 54 (1990), 545-581.

[8] Cockburn, B., Karniadakis, G. E., And Shu, C.-W. Discontinuous Galerkin Methods. Lecture Notes in Computational Science and Engineering. Springer, 2000.

[9] Cockburn, B., Lin, S. Y., And Shu, C. TVB Runge-Kutta local projection discontinuous Galerkin finite element method for conservation laws III: One dimensional systems. J. Comput. Phys. 84 (1989), 90-113.

[10] Cockburn, B., And Shu, C. The Runge-Kutta local projection $p^{1}$-discontinuous Galerkin method for scalar conservation laws. $M^{2}$ AN 25 (1991), 337-361.

[11] COCKBURN, B., AND SHU, C. W. TVB Runge-Kutta local projection discontinuous Galerkin finite element method for conservation laws II: General framework. Math. Comput. 52 (1989), 411-435.

[12] Cockburn, B., And Shu, C. W. The Runge-Kutta discontinuous Galerkin method for conservation laws V: Multidimensional systems. J. Comput. Phys. 141 (1998), 199-224.

[13] Devine, R. B. K., And Flaherty, J. Parallel, adaptive finite element methods for conservation laws. Appl. Numer. Math. 14 (1994), 255-283.

[14] Gassner, G., LÖRChER, F., AND MunZ, C.-D. A contribution to the construction of diffusion fluxes for finite volume and discontinuous Galerkin schemes. J. Comput. Phys. 224, 2 (2007), 1049-1063. 
[15] GAssner, G., LÖRChER, F., AND MunZ, C.-D. A discontinuous Galerkin scheme based on a space-time expansion. II. Viscous flow equations in multi dimensions. $J$. Sci. Comp. 34, 3 (2008), 260-286.

[16] Hartmann, R., And Houston, P. Symmetric interior penalty DG methods for the compressible Navier-Stokes equations I: Method formulation. Int. J. Num. Anal. Model. 3, 1 (2006), 1-20.

[17] Hesthaven, J. A stable penalty method for the compressible Navier-Stokes equations. II. One-dimensional domain decomposition schemes. SIAM J. Sci. Comput. 18 (1998), 658-685.

[18] Hesthaven, J. A stable penalty method for the compressible Navier-Stokes equations. III. Multi dimensional domain decomposition schemes. SIAM J. Sci. Comput. 20 (1999), 62-93.

[19] Hesthaven, J., And Gottlieb, D. A stable penalty method for the compressible Navier-Stokes equations. I. Open boundary conditions. SIAM J. Sci. Comput. 17 (1996), 579-612.

[20] Hesthaven, J., And Gottlieb, D. Stable spectral methods for conservation laws on triangles with unstructured grids. Comput. Methods Appl. Mech. Eng. 175 (1999), $361-381$.

[21] Hesthaven, J., Gottlieb, S., And Gottlieb, D. Spectral Methods for TimeDependent Problems. Cambridge University Press, Cambridge, 2006.

[22] Hesthaven, J., And Teng, C. H. Stable spectral methods on tetrahedral elements. SIAM J. Sci. Comput. 21 (2000), 2352-2380.

[23] Hesthaven, J. S. From electrostatics to almost optimal nodal sets for polynomial interpolation in simplex. SIAM J. Numer. Anal. 35, 2 (1998), 655-676.

[24] Hesthaven, J. S., And Warburton, T. Nodal high-order methods on unstructured grids I: Time-domain solution of Maxwell's equations. J. Comput. Phys. 181, 1 (2002), 186-221.

[25] Hesthaven, J. S., And Warburton, T. Nodal Discontinuous Galerkin Methods: Algorithms, Analysis, and Applications. Springer Verlag, New York, 2008.

[26] Hu, C., AND SHU, C.-W. Weighted essentially non-oscillatory schemes on triangular meshes. J. Comput. Phys. 1505 (1999), 97-127.

[27] Johnson, T., AND Patel, V. Flow past a sphere up to a Reynolds number of 300. J. Fluid. Mech. 378 (1999), 19-70.

[28] Klaij, C., Van der Vegt, J. J. W., and Van der Ven, H. Spacetime discontinuous Galerkin method for the compressible NavierStokes equations. $J$. Comput. Phys. 217, 2 (2006), 589-611.

[29] LI, B. Discontinuous Finite Elements in Fluid Dynamics and Heat Transfer. SpringerVerlag, Berlin, 2006. 
[30] LÖrCher, F., GAssner, G., AND MunZ, C.-D. An explicit discontinuous Galerkin scheme with local time-stepping for general unsteady diffusion equations. accepted $\mathrm{J}$. Comput. Phys. 2008.

[31] LÖrcher, F., Gassner, G., AND MunZ, C.-D. A discontinuous Galerkin scheme based on a space-time expansion. I. Inviscid compressible flow in one space dimension. J. Sci. Comp. 32, 2 (2007), 175-199.

[32] LÖRCHER, F., AND MunZ, C.-D. Lax-Wendroff-type schemes of arbitrary order in several space dimensions. IMA J. Num. Anal. 27 (2007), 593-615.

[33] Nitsche, J. A. Über ein Variationsprinzip zur Lösung von Dirichlet-Problemen bei Verwendung von Teilräumen, die keinen Randbedingungen unterworfen sind. Abh. Math. Sem. Univ. Hamburg 36 (1971), 9-15.

[34] Peraire, J., And Persson, P. The compact discontinuous galerkin (cdg) method for elliptic problems. SIAM J. Sci. Comput.. to appear.

[35] REED, W., AND HILL, T. Triangular mesh methods for the neutron transport equation. Technical Report LA-UR-73-479, Los Alamos Scientific Laboratory, 1973.

[36] Tomboulides, A. Direct and large-eddy simulation of wake flows: Flow past a sphere. $\mathrm{PhD}$ thesis, Princeton University, 1993.

[37] Toro, E. Riemann Solvers and Numerical Methods for Fluid Dynamics. Springer, June 1999.

[38] Yan, J., And Shu, C.-W. A local discontinuous Galerkin method for KdV type equations. SIAM J. Numer. Anal. 40, 2 (2002), 769-791.

[39] YAN, J., AND SHU, C.-W. Local discontinuous Galerkin methods for partial differential equations with higher order derivatives. J. Sci. Comput. 17, 1-4 (2002). 